\title{
Hisoleresearch
}

\section{Konzeptevaluierung der Initiative TECXPORT}

FFG-Geschäftsfallnummer BW000023221

Endbericht an die

FFG Österreichische Forschungsförderungsgesellschaft mbH

Sensengasse 1

1090 Wien

Per E-Mail: tecxport@ffg.at

1. Juli 2021

inspire research Beratungsgesellschaft m.b.H.

Lange Gasse 65/16

1080 Wien

Tel. +4313530451

E-Mail: office@inspire-research.at 


\section{Kurzfassung}

Das Bundesministerium für Klimaschutz, Umwelt, Energie, Mobilität, Innovation und Technologie (BMK) startete im Jahr 2017 die Initiative TECXPORT zur Unterstützung des Technologietransfers und des Technologieexports der österreichischen Wirtschaft. Mit der operativen Durchführung der Initiative hat das BMK die Österreichische Forschungsförderungsgesellschaft (FFG) beauftragt.

In Vorbereitung und Begleitung der TECXPORT-Initiative hatte das BMK Studien in Auftrag gegeben, um die volkswirtschaftlichen Effekte des Technologie-Exports zu ermitteln. Diese Studien bestätigten den substanziellen Beitrag technologieexportierender österreichischer Unternehmen am Gesamtexportumsatz, wobei gleichzeitig nach wie vor Nachholbedarf im EU-Vergleich konstatiert wurde.

\section{Beschreibung der TECXPORT-Initiative}

Für die Umsetzung der TECXPORT-Initiative setzte das BMK in den Jahren 2017 bis 2020 drei Instrumente ein: Mit dem Veranstaltungsformat Austrian Technology Days (ATD) werden österreichische Technologieanbieter in spezifischen Technologiefeldern mit Interessenten und Partnern (insbesondere mit potenziellen Kunden) im Zielland gezielt miteinander vernetzt (Instrument 1). Das BMK setzt Initiativen für die Ausrichtung von ATD strategisch je nach Bedarf im Zielland aufgrund eigener Vorerhebungen oder aufgrund von Hinweisen von Technologie-Attachés und Wirtschaftsdelegierten im Ausland sowie von im Ausland tätigen österreichischen Technologieanbietern. Für die Teilnahme an ATD können österreichische Technologieanbieter Reisekostenzuschüsse beantragen (Instrument 2). Als Unterstützungstool für die Tätigkeiten von Intermediären (das sind vor allem die fachzuständigen Mitarbeiter ${ }^{*} i n n e n$ an den österreichischen Außenhandelsstellen und Botschaften) wurde im Rahmen der Initiative schließlich die TECXPORT-Plattform aufgebaut (Instrument 3). Dabei handelt es sich um eine sowohl online unter www.tecxport.at als auch offline bzw. als mobile App zugängliche Präsentation österreichischer Technologiekompetenzen entlang der Technologieschwerpunkte Energie, Umwelt, Gesundheitsversorgung, Industrie und Raumfahrt, Mobilität, Sicherheit und Verteidigung, Intelligente Städte, Intelligente Lösungen und IKT sowie Transport und Infrastruktur. Die Plattform bietet die Informationen in sechs Sprachen an. Damit soll es den Technologie-Attachés und Wirtschaftsdelegierten ermöglicht werden, zielgerichtet Informationen über österreichische Technologieangebote zu recherchieren, um sie dann beispielsweise an ihre Ansprechpartner*innen im Ausland bzw. potenzielle Kunden weitergeben zu können.

\section{Ziele der Konzeptevaluierung}

Für die Weiterentwicklung der Initiative TECXPORT beauftragte die FFG in Abstimmung mit dem BMK eine Konzeptevaluierung. Ziel der Evaluierung war es, die Konzeption, die bisherige Umsetzung, sowie die zum jetzigen Zeitpunkt bereits feststellbaren Beiträge zur Zielerreichung und gegebenenfalls Wirkungen des Programms zu analysieren. Mit der Evaluierung soll die bisherige Programmumsetzung reflektiert und Schlussfolgerungen bzw. Empfehlungen für die Weiterführung des Programms entwickelt werden. Im Blickpunkt standen dabei die angebotenen TECXPORT-Förderinstrumente sowie deren Einbettung in die österreichische Förder- und Unterstützungslandschaft für Technologieinternationalisierung insgesamt. 
Mit der Evaluierung sollen Aussagen über die Alleinstellungsmerkmale der TECXPORT-Initiative gemacht werden und über spezifische Schwerpunkte und Besonderheiten, die von anderen Förder- und Unterstützungsinstrumenten für die Technologieinternationalisierung nicht abgedeckt werden.

\section{Methoden und Durchführung}

Methodisch basierte die Evaluierung auf einer Analyse der vom BMK und der FFG zur Verfügung gestellten Programmdokumente, einschließlich der Arbeitsberichte und Jahrespläne seit Start der Initiative. Das BMK stellte darüber hinaus Informationen und Dokumente über weitere für die Evaluierung relevante Maßnahmen und Aktivitäten des BMK für die Technologieinternationalisierung zur Verfügung. Zusätzlich bildeten Gespräche mit Vertreter*innen von österreichischen Technologieanbietern, die in den Jahren 2017 bis 2020 an TECXPORTVeranstaltungen teilgenommen hatten, sowie Gespräche mit BMK-Technologieattachés und Wirtschaftsdelegierten im Ausland eine wichtige empirische Grundlage für diese Evaluierung.

\section{Alleinstellungsmerkmale und Wirkungen der Förderinstrumente}

Die Ergebnisse der Konzeptevaluierung zeigen, dass die TECXPORT-Initiative und ihre Instrumente sowohl komplementär zu den BMK-eigenen weiteren Förder- und Unterstützungsangeboten für die Technologie-Internationalisierung als auch zu Angeboten anderer Akteure in der österreichischen Förder- und Unterstützungslandschaft sind.

Das wichtigste Alleinstellungsmerkmal der Austrian Technology Days (ATD) besteht in der Türöffner-Funktion des BMK für die teilnehmenden österreichischen Technologieanbieter. Durch die proaktive Rolle des BMK bei der Vorbereitung, Durchführung und Nachverfolgung der ATD-Veranstaltungen können für die teilnehmenden österreichischen Technologieanbieter in den Zielmärkten effektive Zugangswege zu (staatlichen) Stellen eröffnet werden. Damit werden Kontakte und Netzwerke für die Anbahnung von Technologie-Exportgeschäften angestoßen. Diese proaktive Rolle des BMK ist nach Einschätzung von ATD-Teilnehmer*innen vor allem in Staaten wichtig, in denen die staatlichen Verwaltungen eine starke Rolle im Wirtschaftsgeschehen spielen, bzw. in Wirtschaftssektoren, die durch staatliche Akteure dominiert bzw. maßgeblich mitgestaltet werden. Dies ist vor allem im Bereich der (öffentlichen) Infrastruktur (z. B. Energie, Verkehr, Versorgung) der Fall. Hier sehen die ATD-Teilnehmer*innen auch den wesentlichen Mehrwert im Vergleich zu anderen Veranstaltungen der Außenwirtschaftsförderung, die sich an österreichische Unternehmen richte. Durch das "quasi-offizielle" Format der ATD-Veranstaltungen würden Kontakte zu Schlüsselpersonen und Entscheidungsträger*innen in den Zielländern ermöglicht werden, die ohne BMK-Unterstützung nicht oder nur mit deutlich höherem Aufwand geknüpft hätten werden können.

Reisekosten für die Teilnahme an Auslandsveranstaltungen für die Technologie-Internationalisierung sind nicht nur in der TECXPORT-Initiative sondern zum Teil auch in anderen Förder- und Unterstützungsprogrammen des Bundes und der Länder förderfähig. Die Ergebnisse der Interviews legen zudem nahe, dass bei diesem Förderinstrument nur mit geringen Additionalitätseffekten gerechnet werden kann. Der Reisekostenzuschuss steht auch in einem Spannungsverhältnis zum förderkonzeptionellen Anspruch, durch eine angemessene Eigenbeteiligung der Technologieanbieter an den Kosten, das notwendige strategische Commitment der Teilnehmer*innen zum Ziel der ATD-Veranstaltungen (d. h. konsequentes 
Weiterverfolgen der etablierten Geschäftskontakte zu konkreten Technologieinternationalisierungsprojekten) sicherzustellen. Gleichzeitig können im Einzelfall Reiskostenzuschüsse zweckmäßig sein, z. B. um bei thematisch fokussierten ATD-Veranstaltungen die Teilnahme zentraler österreichischer Technologieanbieter sicherzustellen.

Mit Blick auf die Inhalte, die Ausrichtung und die Funktionalität der TECXPORT-Plattform bestehen keine alternativen oder vergleichbaren Angebote. Die österreichische Außenwirtschaftsagentur bietet für die Präsentation auf Zielmärkten zwar unternehmensbezogene Verzeichnisse an. Mit der technologiebezogenen Darstellung österreichischer Kompetenzen hat die TECXPORT-Plattform ein klares Alleinstellungsmerkmal. Außerdem richtet sich die TECXPORT-Plattform als einziges Angebot explizit an Intermediäre als primäre Zielgruppe. Auch hinsichtlich Funktionalität (online, offline, mobil, webbasiert) und verfügbarer Sprachversionen ist die TECXPORT-Plattform ein besonderes Instrument. Die Interviews bestätigten die Zweckmäßigkeit der strategischen Zielsetzung und das bestehende Potenzial der TECXPORT-Plattform als ein Unterstützungsinstrument für österreichische Intermediäre und ihre Partner im Ausland.

Die Kooperation des BMK mit seinen Partnern ermöglicht es, mit den für TECXPORT zur Verfügung stehenden finanziellen Mittel eine möglichst große Wirkung zu erzielen. Die Strategie des BMK, mit der TECXPORT-Initiative auf definierte Technologieschwerpunkte und Zielmärkte zu setzen, wird durch die zunehmende Bedeutung von Technologie-Internationalisierung innerhalb des Systems der österreichischen Außenwirtschaftsförderung insgesamt bestätigt. In den vergangenen Jahren gab es beispielsweise Bestrebungen, Außenwirtschaft Austria zunehmend als Innovationsagentur zu positionieren. Durch die aktive Mobilisierung der Kompetenzen der FFG für die Technologie-Internationalisierung sowie durch das Design des Veranstaltungsformats ATD und der TECXPORT-Plattform zur Präsentation österreichischer Technologieangebote hat das BMK diese Entwicklung vorweggenommen.

\section{Empfehlungen zur zukünftigen Ausrichtung der TECXPORT-Initiative}

Die Ergebnisse aus den Interviews zu den Aktivitäten, Ergebnissen und Wirkungen lassen den Schluss zu, dass es dem BMK mit den Maßnahmen der TECXPORT-Initiative gelingt, österreichische Technologieanbietern einen einfacheren Zugang in den ausgewählten Zielmärkten zu ermöglichen. Für die zukünftige Ausrichtung der TECXPORT-Instrumente wurden aus den empirischen Ergebnissen der Konzeptevaluierung die folgenden Empfehlungen abgeleitet:

- Beim Instrument ATD sollte das BMK weiterhin sehr klar auf seine Türöffner-Funktion setzen. Es sollte auf die Unterstützung konkreter Bedarfe (österreichischer Technologieanbieter) im Zielland fokussieren, die entweder aktuelle innovations-und technologiepolitische Schwerpunkte des Ministeriums adressieren oder die sich auf die bilateralen ministeriellen Abkommen über die Technologiezusammenarbeit zwischen Österreich und anderen Staaten in internationalen Wachstums- und Zukunftsmärkten beziehen. Die identifizierten Schwerpunkte Energie, Mobilität, Umwelt, Gesundheit, Industrielle Technologien, Luft- und Raumfahrt, IKT und (urbane) Infrastruktur und Sicherheit sollten weiterverfolgt werden.

- Die Gestaltung der Förderung bei Reisekostenzuschüssen für TECXPORT-Veranstaltungen sollte überdacht bzw. neu konzipiert werden. Das BMK sollte das Instrument möglichst strategisch einsetzen und auf Teilnehmer*innen konzentrieren, bei denen eine ad- 
ditionale Wirkung der Förderung am ehesten erwartet werden kann bzw. für Fälle, in denen die Gewährung eines Reisekostenzuschusses einen wesentlichen Beitrag für den Erfolg einer ATD-Veranstaltung leisten kann.

- Das Nutzer- und Nutzungskonzept der TECXPORT-Plattform sollte weiter geschärft und fokussiert werden. Die TECXPORT-Plattform sollte klar als Unterstützungstool für österreichische Intermediäre (Technologie-Attachés, Wirtschaftsdelegierte) positioniert und weiterentwickelt werden. Bereits bestehende Aktivitäten der FFG zur kontinuierlichen Qualitätssicherung des inhaltlichen Angebots und zur stärkeren Bekanntmachung und Nutzung der TECXPORT-Plattform durch Intermediäre sollten weitergeführt bzw. intensiviert werden.

- Die Gespräche mit österreichischen Technologieanbietern wiesen auf einen substanziellen Bedarf nach FTI-Kooperationen mit Partnern in Zielländern zur Vorbereitung und Unterstützung von konkreten Technologieexportprojekten hin. Das BMK und die FFG entwickeln aktuell bereits zusätzliche TECXPORT-Förderinstrumente, um diesem Bedarf nach bilateralen FTI-Kooperationen entgegenzukommen. Bei der Positionierung, Gestaltung und Abwicklung von neuen FTI-Förderangeboten sollte auf eine optimale Einbettung in das bereits bestehende FFG-Förderangebot geachtet werden. Auf bereits bestehende Erfahrungen der FFG mit bilateralen FTI-Ausschreibungen (in den Zielländern) sollte aufgebaut werden.

- Die Instrumente und Verfahren bei zukünftigen FTI-Förderungen sollten auf die zur Verfügung stehenden Förderbudgets abgestimmt sein. Ausschreibungen sollten Wettbewerb zwischen Antragstellern zulassen, gleichzeitig aber auch eine ausreichende Förderwahrscheinlichkeit sicherstellen. Der administrative Aufwand der Förderstelle und der Antragsteller sollten in einem vertretbaren Verhältnis zum Förderbudget bleiben.

- Strategisch sollte das BMK weiter auf Kooperationen setzen, um Synergien mit anderen Akteuren im österreichischen Förder- und Unterstützungssystem für Technologie-Internationalisierung zu sichern. Insbesondere ist es dem BMK gelungen, die Kompetenzen der FFG im Bereich der Forschungs- und Innovationsförderung für die Technologieinternationalisierung insgesamt besser sichtbar und für Dritte nutzbar zu machen (z. B. Außenwirtschaft Austria). Auf diese Dimension des Programms sollte in Zukunft noch stärker geachtet werden.

- Für das Programm und für die einzelnen Förderinstrumente der TECXPORT-Initiative sollten Ziele und Zielindikatoren festgelegt werden, die Aussagen über den Erfolg der Maßnahmen über die bestehenden Monitoringmaßnahmen hinaus zulassen und die mit den Förderinstrumenten auch direkt adressiert werden können. Das strategische und operative Monitoring zur Unterstützung der Governance und der laufenden Programmsteuerung sollte ausgebaut bzw. systematisiert werden. 


\section{Executive Summary}

In 2017, the Federal Ministry of Climate Action, Environment, Energy, Mobility, Innovation and Technology (BMK) launched the TECXPORT initiative to support technology transfer and technology export of the Austrian economy. The BMK has commissioned the Austrian Research Promotion Agency (FFG) with the operational implementation of the initiative.

In preparation for and in support of the TECXPORT initiative, the BMK commissioned studies to determine the economic effects and returns of Austria's technology exports. These studies confirmed the substantial contribution of technology-exporting Austrian companies to total export turnover. At the same time the studies identified a need to catch up in comparison with other EU states.

\section{Description of the TECXPORT initiative}

In the years 2017 to 2020 the BMK used three instruments to implement the TECXPORT initiative: Austrian Technology Days (ATD) is an event format that links and networks Austrian technology providers in specific technology with interested parties and partners (i.e. potential customers) in the target country (instrument 1). The BMK takes the initiative for new ATD strategically on the basis of specific needs in the target country. The information on the needs is gathered via the ministry's own intelligence gathering or on the basis of information coming from technology attachés and business delegates abroad as well as from Austrian technology providers operating abroad. Austrian technology providers can apply for travel grants for participation in ATD (instrument 2). Finally, the TECXPORT platform (Instrument 3) was set up as a support tool for the activities of intermediaries (i.e. staff at the Austrian foreign trade offices and embassies responsible for technology export). The TECXPORT platform presents Austrian technological competences along the technology priorities (energy, environment, healthcare, industry and space, mobility, security and defence, smart cities, smart solutions and ICT, transport and infrastructure) either online (www.tecxport.at) or offline or as a mobile app. The platform offers information in six languages. The aim is to enable technology attachés and business delegates to research information on Austrian technology offers in a targeted manner, so that they can then pass it on to their contacts abroad or to potential customers.

\section{Goals of the concept evaluation}

For the further development of the TECXPORT initiative, FFG commissioned this concept evaluation in coordination with the BMK. The objective of the evaluation was to analyse the concept, the implementation, the contributions to the overall achievement of objectives and, if possible, any effects of the programme that are already visible. The evaluation shall reflect on the implementation of the programme and develop conclusions and recommendations for the further improvement of the programme. The focus of the evaluation is on the TECXPORT funding instruments and their integration into the Austrian funding and support landscape for technology internationalisation as a whole. The evaluation is intended to provide information on the USPs of the TECXPORT initiative and on specific strengths and features that are not covered by other funding and support instruments for technology internationalisation in Austria. 


\section{Methods and implementation}

Methodologically, the evaluation was based on an analysis of the programme documents provided by the BMK and the FFG, including the work reports and annual plans since the start of the initiative. The BMK also provided information and documents on other BMK measures and activities for technology internationalisation that are relevant for this evaluation. In addition, interviews with representatives of Austrian technology providers who had participated in TECXPORT events in the past, as well as interviews with BMK technology attachés and Austrian business delegates abroad, provided the empirical basis for this evaluation.

\section{USPs and effects of the funding instruments}

The results of the concept evaluation show that the TECXPORT initiative and its instruments are both complementary to the BMK's other support offers for technology internationalisation and to the offers provided by other actors in the Austrian support landscape.

The most important USP of the Austrian Technology Days (ATD) is the door-opening function of the BMK for the participating Austrian technology providers. Through the proactive role of the BMK in the preparation, implementation and follow-up of the ATD events, effective access channels to (government) agencies can be opened for the participating Austrian technology providers in the target markets. The established contacts help initiate further activities and technology export business. According to ATD participants, the proactive role of the BMK is particularly important in countries where state administrations play a strong role in economic activity, or in economic sectors that are dominated or significantly shaped by state actors. This is particularly the case in the area of (public) infrastructure (e.g. energy, transport, utilities). This is also where ATD participants see the main added value compared to other foreign trade promotion events aimed at Austrian companies. The "quasi-official" format of the ATD events facilitates contacts with key persons and decision-makers in the target countries that would not have been possible without BMK support, or only with significantly greater effort.

Travel costs for participation in foreign events for technology internationalisation are not only eligible for funding in the TECXPORT initiative, but also in other funding and support programmes of the federal government and on provincial level. The findings from the interviews suggest that only minor additionality effects can be expected with this funding instrument. The travel allowance is also in conflict with the conceptual claim to ensure the necessary strategic commitment of the participants to the goal of the ATD events (i.e. follow-up of the established business contacts which shall lead to successful technology internationalisation projects) which is expressed by covering your own costs for participating in the ATD events. At the same time, travel subsidies may be appropriate in individual cases, e.g. to ensure the participation of key Austrian technology providers in thematically focused ATD events.

With regard to the content, orientation and functionality of the TECXPORT platform, there are no alternative or comparable offers available in Austria. The Austrian trade promotion organisation Außenwirtschaft Austria does offer company-related directories for the presentation on target markets. With the focus on Austrian technological capabilities and competences, the TECXPORT platform has a clear USP. In addition, the TECXPORT platform is the only offer explicitly aimed at intermediaries as the primary target group. The TECXPORT platform is also special in terms of functionality (online, offline, mobile, web-based) and language coverage. The interviews confirmed the appropriateness of the strategic objectives of the TECXPORT platform and its potential as a support tool for intermediaries and their partners abroad. 
The cooperation between the BMK and its partners helps maximise the impact given the limited financial resources available for TECXPORT. The BMK's strategy of focusing with the TECXPORT initiative on defined technology priorities and target markets is in line with the increasing importance of technology internationalisation within the system of Austrian foreign trade promotion in general. In recent years, for example, efforts have been made to increasingly position Außenwirtschaft Austria as an innovation agency. By actively mobilising the competences of the FFG for technology internationalisation and by designing the ATD event format and the TECXPORT platform for presenting Austrian technology offers, the BMK has anticipated this development.

\section{Recommendations for the future direction of the TECXPORT initiative}

The results from the interviews on the activities, outcomes and impacts of the TECXPORT initiative show that the BMK succeeds with the support instruments in providing Austrian technology providers with easier access in the selected target markets. For the future orientation of the TECXPORT instruments, the evaluation gives the following recommendations:

- Concerning Austrian Technology Days (ATD), the BMK should continue to focus on its door-opening function and to provide venues that either address current innovation and technology policy priorities of the ministry, demand expressed by Austrian technology providers, specific needs in target countries or that relate to the bilateral ministerial agreements on technology cooperation between Austria and other countries in international growth and future markets. The thematic priority areas of energy, mobility, environment, health, security, industrial technologies, aerospace, ICT and (urban) infrastructure and security should be continued.

- The funding design for ATD travel grants should be reconsidered or redesigned. The BMK should use the instrument as strategically as possible and concentrate on participants for whom an additional effect of the funding can most likely be expected, or for cases in which the travel grant can make a significant contribution to the success of an ATD event.

- The user and utilisation concept of the TECXPORT platform should be further sharpened and focused. The TECXPORT platform should be clearly positioned and further developed as a support tool for Austrian intermediaries (technology attachés, business delegates). Existing FFG activities for quality assurance of the content and awareness raising and use of the TECXPORT platform among intermediaries should be continued and intensified.

- The interviews with Austrian technology providers indicated a substantial need for RTI cooperation with partners in target countries to prepare and support specific technology export projects. The BMK and the FFG are currently in the process to develop additional TECXPORT funding instruments to meet the demand for bilateral RTI cooperation. When designing and implementing new RTI funding schemes, care should be taken to ensure that they are optimally embedded in the existing FFG funding structure. The FFG has already acquired substantial experience with bilateral RTI calls (in the target countries) which should be built upon.

- The instruments and procedures for future RTI funding schemes should reflect the available funding budgets. Calls for proposals should allow for competition between applicants, but at the same time ensure a sufficient proposal success rate. The administrative 
costs of the funding agency and the applicants should be appropriate with regard to the funding budget available.

- Strategically, the BMK should continue cooperation with partners in order to secure synergies with other actors in the Austrian funding and support system for technology internationalisation. In particular, the BMK has succeeded in visibly positioning the FFG with its competences in research and innovation promotion as an actor and partner for technology internationalisation for third parties (e.g. Außenwirtschaft Austria). More attention should be paid to this dimension of the TECXPORT programme in the future.

- Suitable goals and indicators should be set out for the TECXPORT programme and for the individual funding instruments of the initiative, which allow to assess the achievement of goals and which can be directly related to the funding instruments. Strategic and operational monitoring to support governance and ongoing programme management should be expanded and systematised. 


\section{Inhaltsverzeichnis}

Einleitung. 2

1.1 Hintergrund und Ziele der TECXPORT-Initiative .................................................................

1.2 Ziele, Leitfragen und Durchführung der Konzeptevaluierung ...................................................

2 Einbettung und Alleinstellungsmerkmale ........................................................................................5

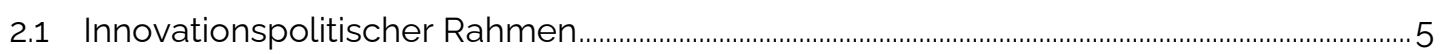

2.2 Förder- und Unterstützungsangebote des BMK für die TechnologieInternationalisierung...........................................................................................................................

2.3 Einbettung und Positionierung in der Förderungs- und Unterstützungslandschaft

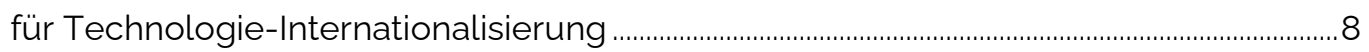

2.4 Alleinstellungsmerkmale der TECXPORT-Initiative ....................................................................... 11

3 Relevanz und Nutzen für die Zielgruppen ..................................................................................

3.1 Austrian Technology Days (ATD) ...................................................................................................15

3.2 ATD-Reisekostenzuschüsse ...........................................................................................................16

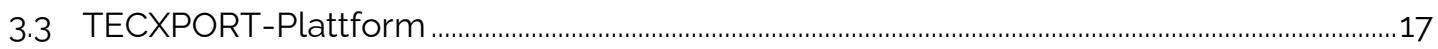

4 Empfehlungen zur zukünftigen Ausrichtung ...................................................................................20

4.1 Empfehlungen zur Weiterentwicklung der Förderinstrumente ..............................................20

4.2 Empfehlungen zur Gestaltung der Prozesse der TECXPORT-Initiative............................... 23 
Die Österreichische Forschungsförderungsgesellschaft (FFG) beauftragte eine Konzeptevaluierung der Initiative TECXPORT. In diesem einleitenden Abschnitt stellen wir die TECXPORT-Initiative und die Ziele der Konzeptevaluierung dar.

\subsection{Hintergrund und Ziele der TECXPORT-Initiative}

Das Bundesministerium für Klimaschutz, Umwelt, Energie, Mobilität, Innovation und Technologie (BMK, ehemals Bundesministerium für Verkehr, Innovation und Technologie - BMVIT) startete im Jahr 2017 die Initiative TECXPORT zur Unterstützung des Technologietransfers und des Technologieexports der österreichischen Wirtschaft. In Vorbereitung und Begleitung der TECXPORT-Initiative hatte das BMK Studien in Auftrag gegeben, um die volkswirtschaftlichen Effekte des Technologie-Exports zu ermitteln. Diese Studien bestätigten den substanziellen Beitrag technologieexportierender österreichischer Unternehmen am Gesamtexportumsatz, wobei gleichzeitig nach wie vor Nachholbedarf im EU-Vergleich konstatiert wurde. ${ }^{1}$

Ausgangspunkt für die Initiative ist damit die große gesamtwirtschaftliche Bedeutung des Technologieexports für die österreichische Wirtschaft. Mit der Initiative TECXPORT unterstützt das BMK durch eine gezielte Positionierung österreichischer Technologie im Ausland die Verbesserung der Wettbewerbsposition österreichischer Unternehmen. Mit der weltweiten Vermarktung von Technologie-Innovationen "Made in Austria" soll die österreichische Wirtschaft gefördert werden. Langfristig sollen die Schwerpunkte und Maßnahmen der TECXPORT-Initiative dazu beitragen, den Technologieanteil im Export und die Anzahl technologieexportierender österreichischer Unternehmen zu steigern sowie die Wirkung der nationalen anwendungsorientierten Forschungsförderung zu verbessern.

In den Jahren 2017 bis 2020 erfolgte die Finanzierung der Initiative unter der Schirmherrschaft des BMK aus Mitteln des Österreich-Fonds. Seit 2021 finanziert das BMK die Initiative aus eigenen Budgetmitteln. Für die operative Durchführung der Initiative hat das BMK die Österreichische Forschungsförderungsgesellschaft (FFG) beauftragt, die für die Umsetzung der Maßnahmen mit Außenwirtschaft Austria, der Internationalisierungs- und Innovationsagentur der österreichischen Wirtschaft der Wirtschaftskammer Österreich (WKÖ) zusammenarbeitet.

Die primäre Zielgruppe der TECXPORT-Initiative sind die österreichischen Technologieanbieter. Es werden dabei sowohl produzierende Unternehmen und gewerbliche Dienstleistungsunternehmen als auch anwendungsorientierte Forschungseinrichtungen angesprochen, die über entsprechende Technologieangebote verfügen. Die zweite Zielgruppe der TECXPORTInitiative sind intermediäre Akteure der Technologieexportlandschaft, insbesondere die österreichischen Wirtschaftsdelegierten im Ausland, die Mitarbeiter*innen anderer Ministerien an den österreichischen Botschaften und Auslandsvertretungen sowie die Technologie-Attachés des BMK an Auslandsvertretungen. Diese Personengruppen sollen mit den TECXPORT-Angeboten unterstützt werden, österreichische Technologiekompetenzen im

\footnotetext{
${ }^{1}$ Berrer, H. et al., 2021, Wirtschaftsmotor Technologie-Export. Studie zur Ermittlung der volkswirtschaftlichen Effekte des Technologie-Exports. Studie im Auftrag des Bundesministeriums für Klimaschutz, Umwelt, Energie, Mobilität, Innovation und Technologie. Economica Institut für Wirtschaftsforschung, März 2021, Wien.
} 
Ausland bekannt zu machen und potenzielle Kunden mit österreichischen Technologieanbietern zu vernetzen.

Das BMK fokussiert seine Maßnahmen auf die Technologiefelder Energie, Umwelt, Gesundheitsversorgung, Industrie und Raumfahrt, Mobilität, Sicherheit und Verteidigung, Intelligente Städte, Intelligente Lösungen und IKT sowie Transport und Infrastruktur. Das BMK legt damit die Schwerpunkte vor allem in Technologiefeldern, für die das Ministerium sachlich auch zuständig ist. Ausgangspunkt für länderbezogene Maßnahmen im Rahmen der TECXPORT-Initiative sind häufig die bilateralen Abkommen Österreichs mit anderen Staaten über die Intensivierung der technologischen Zusammenarbeit in den genannten Technologiefeldern. Das BMK wird insbesondere dann aktiv, wenn seitens der Zielländer ein besonderer Bedarf nach Technologielösungen artikuliert wird, die von österreichischen Technologieanbietern zur Verfügung gestellt werden können, bzw. wenn anzunehmen ist, dass österreichische Technologieanbieter durch ein proaktives Engagement des BMK leichter Zugang zu wichtigen Zukunfts- und Wachstumsmärkten erhalten könnten.

Die Aktivitäten des BMK sollen komplementär zu den Maßnahmen anderer Akteure ausgerichtet sein, um die Effektivität und Effizienz des österreichischen Gesamtsystems der Technologieexportförderung insgesamt weiter zu steigern.

\subsection{Ziele, Leitfragen und Durchführung der Konzeptevaluierung}

Diese Evaluierung beschäftigt sich mit der Konzeption, der bisherigen Umsetzung, sowie mit den bereits feststellbaren Wirkungen und Beiträgen zur Erreichung der Ziele der Initiative. Die Evaluierung ist als Konzeptevaluierung ausgerichtet. Die Evaluierung analysiert dazu die angebotenen TECXPORT-Instrumente (tecxport.at-Plattform, Austrian Technology Days, Reisekostenzuschüsse) hinsichtlich ihrer Alleinstellungsmerkmale und ihrer Einbettung in die österreichische Förder- und Unterstützungslandschaft für Technologie-Internationalisierung. Die Evaluierung reflektiert darüber hinaus den bisherigen Programmverlauf sowie die intern diskutierten Ansätze zur Erweiterung der angebotenen Instrumente. Auf Grundlage dieser Analysen leitet die Evaluierung Schlussfolgerungen ab und entwickelt Empfehlungen, die als Grundlage für die Weiterentwicklung von TECXPORT dienen können.

Im Zentrum der Konzeptevaluierung stehen die folgenden Fragen:

\section{Zum Konzept der TECXPORT-Initiative}

- Wie relevant ist das TECXPORT-Angebot für die Zielgruppen?

- Wie ist das TECXPORT-Angebot in die nationale Förder- und Unterstützungslandschaft für Technologie-Internationalisierung eingebettet?

- Was sind die Alleinstellungsmerkmale der TECXPORT-Angebote? Welche Besonderheiten weist TECXPORT auf, die von anderen, vergleichbaren Programmen nicht abgedeckt werden?

- Können mit der budgetären Ausstattung der TECXPORT-Initiative die angestrebten Ziele angemessen adressiert werden?

Zu den Ergebnissen und Wirkungen der TECXPORT-Initiative:

- Wie wird TECXPORT von den Zielgruppen angenommen? 
- Welchen Nutzen hatte die Online-Plattform und die Austrian Technology Days bisher für die Umsetzung konkreter Vorhaben (z. B. Exportprojekte) bei den Zielgruppen?

- Lässt der bisherige Verlauf der Initiative sowie die beobachteten Aktivitäten, Ergebnisse und Wirkungen bei den Zielgruppen erwarten, dass die TECXPORT-Initiative ihre Ziele erreicht?

- Welche zusammenfassenden Empfehlungen lassen sich aus den Ergebnissen der Evaluierung für die künftige Ausrichtung der TECXPORT-Initiative ableiten? Könnten die Angebote an die Zielgruppen durch zusätzliche Instrumente verbessert werden?

Um diese Fragen zu beantworten führten wir im April und Mai 2021 eine Auswertung der uns vom BMK und der FFG zur Verfügung gestellten Programmdokumente durch. Darüber hinaus sammelten und analysierten wir Daten und Informationen zum österreichischen Technologieexport und zu vergleichbaren Förderprogrammen und Unterstützungsangeboten bei den Einrichtungen Austria Wirtschaftsservice (aws), Wirtschaftskammer Österreich (WKÖ), Statistik Austria und Österreichische Kontrollbank (OeKB).

Eine zentrale Informationsquelle für die Konzeptevaluierung bildeten außerdem persönliche, telefonische Interviews, die wir mit den Programm verantwortlichen Personen im BMK, mit Vertreter*innen des TECXPORT-Teams der FFG, mit den BMK-Vertretern in den Technologie-Büros in Peking und Jakarta, mit dem Wirtschaftsdelegierten für Hongkong sowie mit Vertreter*innen von österreichischen Technologieanbietern, die in der Vergangenheit bereits an TECXPORT-Maßnahmen beteiligt waren bzw. über TECXPORT-Maßnahmen Kontakte in Zielmärkte knüpfen konnten, führten. 


\section{Einbettung und Alleinstellungsmerkmale}

In diesem Abschnitt stellen wir die Einbettung der TECXPORT-Initiative in die Förder- und Unterstützungslandschaft für Technologieinternationalisierung in Österreich dar. Wir geben einen Überblick über die zentralen Akteure und ihre Unterstützungsangebote. Im Anschluss daran diskutieren wir die Alleinstellungsmerkmale der TECXPORT-Förderinstrumente.

\subsection{Innovationspolitischer Rahmen}

Die TECXPORT-Initiative leistet einen Beitrag zur Umsetzung des aktuellen Regierungsprogramms sowie mehrerer Strategien der österreichischen Bundesregierung. Im Detail adressiert die TECXPORT-Initiative die folgenden Politikschwerpunkte:

- Regierungsprogramm $2020-2024$

- Österreichs Wirtschaft international positionieren: Umsetzung und Ergänzung der bestehenden Außenwirtschaftsstrategie anhand der Schwerpunkte des Regierungsprogramms (Seite 63).

- Technologieoffensive, Digitalisierung und Innovation: Internationale Positionierung Österreichs als Vorreiter im Bereich der erneuerbaren Energie als Unterstützung der österreichischen Exportwirtschaft (Seite 82).

- FTI-Pakt 2021-2023

- Handlungsfeld 1.2.3: FTI zur Erreichung der Klimaziele: Maßnahme: Erhöhung des Impacts von klima- und umweltschutzrelevanten Technologien durch [...] Technologietransfer und -exporte (Technologie-Internationalisierung) [.... .

- Außenwirtschaftsstrategie der österreichischen Bundesregierung

- Maßnahme 46: Internationalisierung der FTI stärken: Durch eine Internationalisierung im Bereich Forschung, Technologie und Innovation (FTI) wäre die angewandte Forschung als Türöffner und zur Positionierung österreichischer Unternehmen in definierten Zielländern einzusetzen [...].

- Maßnahme 56: Bilaterale Kooperationsvereinbarungen nützen: Österreichische Unternehmen werden durch den Abschluss innerstaatlich koordinierter bilateraler Kooperationsvereinbarungen auf Ressortebene durch die zuständigen Ressorts [...] gezielt unterstützt. Dieses Instrument soll zielgerichtet länderspezifisch und branchenspezifisch eingesetzt werden [...].

- Maßnahme 58: Außenwirtschaftliche Förderungen optimieren: [...] Good Practice Projekte wie [...] TECXPORT werden zielgruppenadäquat weiterentwickelt.

- Masterplan Umwelttechnologie

- Maßnahme 8: Exportinitiative Umwelttechnik mit Bezug zu den Detailmaßnahmen: 1) Definition prioritärer Märkte und Länder; 2) Erarbeitung einer Kompetenzlandkarte Umwelttechnik; 3) Präsentation von Referenzprojekten; 4) Schwerpunktsetzung auf Umwelttechnologie bei Auslandsaktivitäten; 5) Sichtbarmachung von Aktivitäten.

- Maßnahme 9: Forcierung der Länder- und Firmenkooperationen mit Bezug zu den Detailmaßnahmen: 1) Starke Vernetzung; 2) Projektpartnerbörse. 
- Maßnahme 10: Ausbau der Förderungsprogramme für Aktivitäten im Ausland mit Bezug zu den Detailmaßnahmen: 2) Unterstützung der Aufbereitung von Märkten; 3) Förderung von Unternehmen am Auslandsmarkt.

\subsection{Förder- und Unterstützungsangebote des BMK für die Technologie-In- ternationalisierung}

Für die Umsetzung der oben genannten Strategien und Schwerpunkte der Bundesregierung setzt das BMK auf ein Set komplementärer Maßnahmen, die sowohl im zwischenstaatlichen Bereich als auch bei konkreten Förder- und Unterstützungsangeboten für österreichische Technologieanbieter ansetzen:

\section{Bilaterale Abkommen zur technologischen Zusammenarbeit}

Auf zwischenstaatlich-politischer Ebene verfolgt die BMK Stabsstelle Technologietransfer die Vorbereitung, den Abschluss und die Implementierung von bilateralen Abkommen über die Intensivierung der technologischen Zusammenarbeit in bestimmten Themenfeldern. Das BMK nutzt diese bilateralen Abkommen strategisch, um die Technologieinternationalisierung österreichischer Anbieter zu unterstützen. Mit Ende 2020 bestanden rund 120 bilaterale Abkommen mit 55 verschiedenen Staaten.

\section{Technologie-Attachés des BMK an österreichischen Botschaften im Ausland}

Außerdem verfügt das BMK über Technologie-Attachés an österreichischen Botschaften im Ausland (derzeit in China und Indonesien), die aufgrund ihrer örtlichen Nähe zu politischen Entscheidungsträgern im Ausland eine wichtige Mittlerfunktion zwischen dem BMK und den politischen Akteuren bzw. den Kunden auf den Zielmärkten wahrnehmen.

Internationale Marktstudien, FTO-Analysen und Förderung von Demonstrationsanlagen (TECTRANS)

Das Programm TECTRANS ist ein zur TECXPORT-Initiative komplementärer Baustein des BMK für die Förderung der Internationalisierung österreichischer Technologieanbieter. TECTRANS wird operativ durch die aws betreut und umfasst drei Förderinstrumente: Mit dem Modul Internationale Marktstudien können Studien finanziert werden, die aufzeigen, wie österreichische Technologie im internationalen Wettbewerb bei Kunden im Zielland nachhaltig verankert werden kann. Mit dem Modul Internationale Markstudien können außerdem die Entwicklung von Marktauftritts- und Markteintrittsstrategien von Unternehmen in Auslandsmärkten gefördert werden. Mit dem zweiten TECTRANS-Modul Internationale FTO können Freedom-to-Operate (FTO)-Analysen für neue Auslandsmärkten gefördert werden. Darunter fallen beispielsweise Durchsetzungs- oder Verteidigungsstrategien für das geistige Eigentum von österreichischen Technologieanbietern. Gefördert werden können aber auch die Kosten für die Erlangung des Schutzes von geistigem Eigentum für innovative österreichische Technologie auf konkreten Zielmärkten sowie die dazu notwendigen Dienstleistungen. Als drittes Modul bietet TECTRANS Förderungen für Demonstrationsanlagen an, die der internationalen Vermarktung neuer Technologien dienen. Die Anlagen können dabei sowohl im Ausland als auch im Inland errichtet werden. Für die Demonstrationsanlagen können auch damit in Zusammenhang stehende Forschungs- und Entwicklungsvorhaben und Produktadaptierungen gefördert werden. 
Austrian Technology Days (ATD), ATD-Reisekostenzuschüsse und TECXPORT-Plattform (Initiative TECXPORT)

Das Konzept und die Förderinstrumente der BMK Initiative TECXPORT stehen im Zentrum dieser Evaluation. Mit dem Start des Programms wurden auf Grundlage des Programmkonzept die geeigneten Maßnahmen entwickelt. In den Jahren 2017 bis 2020 wurden für die Umsetzung der TECXPORT-Initiative schließlich drei Förderinstrumente eingesetzt:

\section{Austrian Technology Days (ATD)}

Austrian Technology Days (ATD) sind Veranstaltungen im Ausland, bei denen österreichische Technologieanbieter und Interessenten im Zielland gezielt zusammengeführt und vernetzt werden. ATD werden durch die BMK Stabsstelle Technologietransfer zusammen mit den Technologie-Attachés des BMK im Ausland sowie den Kooperationspartnern an den Außenwirtschaftszentren in den jeweiligen Zielländern vorbereitet und auf bestimmte Themen eingegrenzt. Österreichische Technologieanbieter haben die Möglichkeit sich für diese internationalen Networking-Events anzumelden. Bei der Vorbereitung der Veranstaltungen achtet das BMK darauf, jene österreichischen Anbieter mit an Bord zu haben, die für den Bedarf im Zielland mit Blick auf die bestehenden Technologiekompetenzen am besten geeignet sind. Die gemeinsam mit Partnern im Ausland vor Ort oder virtuell durchgeführten Veranstaltungen ermöglichen so den gezielten Austausch zwischen potentiellen Kunden im Zielland und kompetenten Technologieanbietern in Österreich.

In den Jahren 2017 bis 2020 fanden 16 Austrian Technology Days statt, in deren Rahmen sich mehr als 160 Technologieanbieter aus Österreich präsentierten. Die Veranstaltungen in den Jahren 2017 bis 2020 fanden in den Schwerpunktländern Volksrepublik China, Sonderverwaltungszone Hongkong, Taiwan, Indonesien und Russische Föderation statt.

\section{Reisekostenzuschüsse für die an ATD teilnehmenden Einrichtungen}

Technologieanbieter, die an einer Austrian Technology Days-Veranstaltung im Ausland teilnehmen, können Reisekostenzuschüsse in Höhe von bis zu 4.500 Euro beantragen. Die Höhe des Reisekostenzuschusses wird jeweils individuell an den Ort und die Gegebenheiten der jeweiligen ATD angepasst. Bisher wurden von österreichischen Teilnehmer*innen an den ATD der Jahre 2017 bis 2020 rund 50 Reisekostenzuschüsse beantragt.

\section{Online-Plattform für österreichische Technologieangebote (www.tecxport.at)}

Auf der Online-Plattform werden österreichische Technologiekompetenzen entlang der BMK-Technologiefelder (siehe Abschnitt 1.1) auf eine ansprechende und kompakte Weise in verschiedenen Versionen (d. h. Desktop, Mobil, App und Offline Version) sowie in mehreren Sprachen dargestellt und so österreichische Produkte und Services präsentiert. Die Plattform wurde im August 2019 erstmals bei den Technologiegesprächen Alpbach vorgestellt.

Die Plattform richtet sich insbesondere an Intermediäre wie die österreichischen Technologie-Attachés und die Wirtschaftsdelegierten im Ausland sowie an deren Ansprech- und Kontaktpersonen in den Zielländern. Die Plattform ist auch offline verfügbar und soll die Intermediäre dabei unterstützen, zielgerichtet Technologien für Kontakte mit potentiellen Interessenten im Ausland zu finden. Damit sollen österreichische Unternehmen mit Partnern und Kunden im Ausland gezielt vernetzt und der Markteinstieg der Unternehmen in einem neuen Exportmarkt erleichtert werden. 
Die österreichischen Unternehmen füllen für die Teilnahme an der TECXPORT-Plattform ein Technologieprofil aus, das von der FFG bzw. externen Redakteur ${ }^{*} i n n e n$ vor Veröffentlichung inhaltlich bearbeitet und qualitätsgesichert wird. Das Technologieprofil wird auf der Plattform in den sechs Sprachen Deutsch, Englisch, Französisch, Spanisch, Russisch und Chinesisch veröffentlicht. Die Teilnahme ist für die österreichischen Interessenten kostenlos. Derzeit befinden sich ca. 500 Technologieprofile auf der TECXPORT-Plattform.

\section{Weitere geplante Förder- und Unterstützungsinstrumente}

Aktuell arbeiten das BMK und die FFG an der Weiterentwicklung der TECXPORT-Initiative. Ziel der Neuausrichtung ist es, die bisher bestehenden Instrumente besser zu integrieren und zu bündeln, um dadurch eine bessere Sichtbarkeit des Angebots zu erreichen. Außerdem soll die Governance der Initiative vereinfacht werden. Schließlich sind auch zusätzliche Maßnahmen in Vorbereitung, mit deren Hilfe die anwendungsorientierte öffentliche Forschungs-, Entwicklungs- und Innovationsförderung (FTI-Förderung) noch effektiver für die Technologie-Internationalisierung österreichischer Unternehmen genutzt werden soll. Folgende zusätzliche TECXPORT-Instrumente werden derzeit konzipiert:

\section{Bilaterale, internationalisierungsorientierte FTI-Ausschreibungen}

Gemeinsam mit Partnern aus dem Ausland (z. B. Ministerien, Förderagenturen, öffentliche Einrichtungen und Verbände) sollen mit diesem neuen TECXPORT-Instrument Ausschreibungen für bilaterale, vermarktungsfokussierte FTI-Kooperationen durchgeführt werden. Im Zentrum der Ausschreibungen soll die Förderung von bilateralen FTI-Projekten stehen, die der gezielten Positionierung österreichischer Technologieanbieter in ausgewählten Zielländern dienen. Die Vorhaben sollen die beihilferechtlichen Vorgaben für eine FuE-Förderung erfüllen und gleichzeitig bereits im Rahmen des Vorhabenplans die Verwertung der erzielten Projektergebnisse durch einen österreichischen Technologieanbieter auf einem Auslandsmarkt schlüssig aufzeigen. In den unterstützten FTI-Projekten sollen durch regionale Kooperationen neue Märkte für österreichische Technologieanbieter aufbereitet werden.

\section{Instrument: Tailored Innovation für die Technologie-Internationalisierung}

Ein weiteres, derzeit in der Konzeptphase stehendes Instrument, trägt den Arbeitstitel Tailored Innovation. Mit diesem Instrument sollen ergänzend zu den geplanten bilateralen FTIAusschreibungen im Rahmen der TECXPORT-Initiative Förderungen für Unternehmen ermöglicht werden, die FTI-basierte Anpassungsinnovationen für ihre Produkte vornehmen wollen. Dieses Instrument geht davon aus, dass österreichische Technologieanbieter marktspezifische Anpassungen an bereits bestehenden Produkten und Lösungen vornehmen müssen, um sie auf neuen Märkten erfolgreich positionieren zu können. Für diese Entwicklung der marktspezifischen Anpassungen können FTI-Projekte notwendig sein, die durch das TECXPORT-Instrument Tailored Innovation gefördert werden sollen.

\subsection{Einbettung und Positionierung in der Förderungs- und Unterstützungs- landschaft für Technologie-Internationalisierung}

Das BMK setzt die Ziele und Schwerpunkte der TECXPORT-Initiative im Einklang mit den eigenen Prioritäten innerhalb des in Abschnitt 2.1 dargestellten innovationspolitischen Rahmens. Die TECXPORT-Angebote des BMK sollen komplementär zu den Unterstützungsan- 
geboten im eigenen Zuständigkeitsbereich (z. B. TECTRANS) und zu jenen der anderen politisch zuständigen Ministerien sein. Auf operativer Ebene ist das BMK bestrebt mit anderen Akteuren der Außenwirtschaftsförderung bzw. der Technologie-Internationalisierung zusammenzuarbeiten, um die erzielten Wirkungen mit den gesetzten Maßnahmen zu maximieren. Das TECXPORT-Konzept nimmt auf die folgenden Akteure insbesondere Bezug:

Akteure in der Förder- und Unterstützungslandschaft für Technologie-Internationalisierung

Neben dem BMK sind es vor allem das Bundesministerium für Digitalisierung und Wirtschaftsstandort (BMDW), das Bundesministerium für europäische und internationale Angelegenheiten (BMEIA) mit den Auslandsvertretungen Österreichs sowie das Bundesministerium für Finanzen (BMF) für die Gestaltung der rechtlichen Rahmenbedingungen von Instrumenten der Exportfinanzierung, die zentrale Rollen für die Förderung der internationalen Vermarktung von österreichischen Technologieangeboten spielen.

Operativ sind vor allem die Wirtschaftskammer Österreich (WKÖ) mit ihrer Internationalisierungs- und Innovationsagentur Außenwirtschaft Austria (AA) sowie die Oesterreichische Kontrollbank (OeKB) mit ihren Angeboten zur Absicherung und Finanzierung von Exportgeschäften (Kredite, Bürgschaften, Garantien) die wichtigsten Ansprechpartner für technologieexportorientierte österreichische Unternehmen, die Unterstützung suchen.

Weitere Akteure in der Förderungs- und Unterstützungslandschaft für Technologie-Internationalisierung sind die Austrian Development Agency (ADA) vor allem durch Beratung, Vernetzung, Kofinanzierung von Projektkosten und Finanzierung von Machbarkeitsstudien in Entwicklungsprojekten österreichischer Unternehmen, die Oesterreichische Entwicklungsbank (OeEB) vor allem durch Sektorstudien, Marktanalysen und Finanzierung von Projekten in Entwicklungsländern und Emerging Markets, Austria Wirtschaftsservice (aws) vor allem durch Finanzierung und Absicherung von Direktinvestitionen im Ausland, Technologieinternationalisierung von KMU und Studien für den kommerziellen internationalen Technologietransfer sowie nicht zuletzt die Österreichische Forschungsförderungsgesellschaft (FFG) vor allem durch die Förderung von anwendungsorientierter und wirtschaftsnaher Forschung und Unterstützung österreichischer Unternehmen bei der Sondierung und Durchführung von internationalen Kooperationen in Forschung, Entwicklung und Innovation. Gemeinsam mit aws unterhält die FFG auch das Global Incubator Network Austria (GIN), mit dem das österreichische Startup-Ökosystem mit österreichischen und internationalen Startups, Investor ${ }^{*} i n n e n$, Inkubatoren und Akzeleratoren in Israel, China, Singapur, Japan und Südkorea vernetzt werden soll.

Einige Bundesländer bzw. ihre Wirtschaftsförderagenturen bieten im Rahmen von Programmen zur Internationalisierung der Wirtschaft den im Bundesland ansässigen Betrieben Unterstützungsangebote an, die zum Teil aus Mitteln der Europäischen Union kofinanziert werden. Bundesländer können beispielsweise Vorhaben von ansässigen Unternehmen (meist KMU) fördern, durch die deren internationale Ausrichtung verbessert werden soll. Im Fokus der Angebote von Bundesländern stehen häufig Vorhaben, die die Aufnahme oder Neuorientierung von internationalen Geschäftsbeziehungen oder die Erschließung neuer Märkte der Unternehmen zum Ziel haben. Mit den gewährten finanziellen Zuschüssen können je nach Bundesland z. B. Marketingmaßnahmen oder Beratungsleistungen in Zusammenhang mit der Internationalisierung oder die Teilnahme an internationalen Fachmessen und Fachausstellungen außerhalb Österreichs oder die Erstellung von Publikationen, die der Interna- 
tionalisierung dienen oder notwendige Zertifizierungen bzw. Standardisierungen in den Zielländern, die zur Markteinführung des Produktes erforderlich sind, gefördert werden. Schließlich stehen österreichischen Unternehmen verschiedene Aktionsprogramme der EU zur Verfügung, durch die grenzüberschreitende Projekte in den Bereichen Forschung, Entwicklung und Innovation, Bildung, Kreativwirtschaft, Mobilität von jungen Unternehmen sowie für die Erschließung von Märkten im asiatischen Raum unterstützt werden können.

\section{Das Programm go-international}

go-international ist das Internationalisierungsprogramm des BMDW, das seit 2003 gemeinsam mit der WKÖ durchgeführt wird. go-international bietet österreichischen Unternehmen ein breites Veranstaltungsprogramm für den Eintritt in Wachstumsmärkte an. Die Auslandsveranstaltungen haben häufig einen Business-to-Business (B2B)-Fokus und sollen damit die heimischen Unternehmen bei der Partner- und Kundengewinnung und bei der Positionierung im Zielmarkt unterstützen. Neben den Veranstaltungen im Ausland bietet go-international auch Inlandsveranstaltungen an, um Unternehmen zu Strategien, Geschäftschancen und Rahmenbedingungen auf internationalen Zukunftsmärkten zu beraten.

Außerdem können österreichische Unternehmen über go-international mehrere Formen von Direktförderungen für den Eintritt in neue Märkte in Anspruch nehmen. Der Internationalisierungscheck fördert erstmalige Markteintrittskosten (z. B. Marketing, Beratung, Reisen, Veranstaltungen, Inkubatorbüro). Mit dem Bildungsscheck werden externe Weiterbildungsmaßnahmen für Mitarbeiter ${ }^{*} i n n e n$ an Auslandsniederlassungen österreichischer Unternehmen gefördert. Der Digital-Marketing Scheck fördert die externen Kosten für den Aufbau von digitalen Auftritten österreichischer Unternehmen auf Auslandsmärkten. Schließlich können Unternehmen mit dem Projektgeschäft-Scheck eine Teilfinanzierung für ihre externen Kosten zum Aufbau von Know-how für das internationale Projektgeschäft (z. B. Schreiben von Anträgen, externe Datenrecherchen, Vorstudien, Machbarkeitsstudien, Weiterbildung, Marketing, Reisen und Veranstaltungen) erhalten. Die Förderungen stehen KMU - und eingeschränkt auf bestimmte Märkte auch Großunternehmen - offen, sofern durch die Vorhaben eine substanzielle Wertschöpfung in Österreich gesichert ist und die Förderungen beihilferechtlich möglich sind (De-Minimis-Beihilfe).

\section{Finanzierung von Exportgeschäften zur Technologie-Internationalisierung}

Neben den oben beschriebenen Förderungen für die Vorbereitung eines erfolgreichen Marktzugangs für österreichische Technologieanbieter bilden die Angebote zur Finanzierung von konkreten Exportgeschäften die zweite zentrale Säule in der Unterstützungslandschaft für Technologie-Internationalisierung. Für diese Unterstützungsmaßnahmen ist die Oesterreichische Kontrollbank (OeKB) der wichtigste Akteur. Das OeKB-Angebot an Export Services kann in vier Bereiche gegliedert werden und umfasst 1) die Finanzierung von Betriebsmitteln für Exportgeschäfte durch günstige Rahmenkredite in Zusammenarbeit mit den Hausbanken der Unternehmen, 2) das Absichern und Finanzieren von Exporten, 3) das Absichern und Finanzieren von Investitionen und Beteiligungen sowie 4) internationale Finanzierungskooperationen (z. B. bei internationalen Großprojekten).

Die OeKB bietet zudem weitere, beispielsweise exportprojektvorbereitende Unterstützungsinstrumente an. So wickelt die OeKB für das BMF das Soft Loan-Projektvorbereitungsprogramm ab, in dessen Rahmen Machbarkeitsstudien oder Studien finanziert werden können, die sektorspezifische technische Lösungen zum Thema haben, die im Vorfeld eines möglichen Exportprojektes in Entwicklungs- und Schwellenländern notwendig sind. 


\subsection{Alleinstellungsmerkmale der TECXPORT-Initiative}

Mit den TECXPORT-Schwerpunkten zur Technologie-Internationalisierung leistet das BMK einen Beitrag zur Umsetzung der Außenwirtschaftsstrategie der Bundesregierung. Die BMKMaßnahmen sind dabei komplementär zu den Förder- und Unterstützungsmaßnahmen anderer Akteure gestaltet. Das BMK setzt gleichzeitig bewusst auf das Konzept "Synergien durch Kooperation": Durch die Zusammenarbeit mit Außenwirtschaft Austria bei der Umsetzung der TECXPORT-Initiative erreicht das BMK beispielsweise eine möglichst große nationale und internationale Sichtbarkeit seiner Angebote. Die Kooperation erlaubt es dem BMK, die Ressourcen und das Netzwerk der über 100 AußenwirtschaftsCenters (ACS) und Außenwirtschaftsbüros für die Umsetzung der eigenen aktuellen Schwerpunkte und Maßnahmen mitzunutzen. Die Kooperation des BMK mit seinen Partnern ermöglicht es, mit den für TECXPORT zur Verfügung stehenden finanziellen Mittel eine möglichst große Wirkung zu erzielen.

Die Strategie des BMK, mit der TECXPORT-Initiative auf definierte Technologieschwerpunkte und Zielmärkte zu setzen, wird durch die zunehmende Bedeutung von Technologie-Internationalisierung innerhalb des Systems der österreichischen Außenwirtschaftsförderung insgesamt bestätigt. In den vergangenen Jahren gab es beispielsweise Bestrebungen, Außenwirtschaft Austria zunehmend als Innovationsagentur zu positionieren. Durch die aktive Mobilisierung der Kompetenzen der FFG für die Technologie-Internationalisierung sowie durch das Design des Veranstaltungsformats ATD und der TECXPORT-Plattform zur Präsentation österreichischer Technologieangebote hat das BMK diese Entwicklung vorweggenommen.

\section{Austrian Technology Days (ATD)}

Die Austrian Technology Days werden von BMK, FFG und Außenwirtschaft Austria gemeinsam beworben bzw. in Kooperation mit den vorhandenen Einrichtungen des BMK, der AuBenwirtschaft Austria in den Zielländern geplant und durchgeführt. Konzeptionell und strategisch sind die Angebote des BMK und der Außenwirtschaft Austria komplementär gestaltet bzw. ergänzen einander. In der Präsentation des Veranstaltungsangebots der Außenwirtschaft Austria werden ATD als eines der Formate gleichrangig dargestellt. Für interessierte Unternehmen und Technologieanbieter wird damit der Zugang zu Informationen über das gesamte Veranstaltungsprogramm der österreichischen Außenwirtschaftsförderung in den jeweiligen Zielländern - unabhängig vom Träger bzw. Organisator der jeweiligen Veranstaltung - gewährleistet.

Die Rückmeldungen von österreichischen Teilnehmer*innen an ATD bestätigen, dass ATD tatsächlich einen Mehrwert gegenüber anderen Veranstaltungsangeboten der Außenwirtschaft Austria leisten können. Die österreichischen Teilnehmer*innen nehmen dabei weniger die Ausrichtung der ATD auf spezifizierte Themen oder Technologiefelder als Alleinstellungsmerkmal wahr, als vielmehr die besondere Rolle, die dem BMK als Türöffner zu Schlüsselpersonen in den öffentlichen Verwaltungen bzw. zu Entscheidungsträgern in den Zielländern zukommt.

Durch die Initiative und durch die unmittelbare Involvierung des BMK in die Vorbereitung, Durchführung und Nachbereitung der ATD-Veranstaltungen können auf den Zielmärkten Türen zu jenen (staatlichen) Stellen geöffnet werden, die Voraussetzung dafür sind, um Kontakte und Netzwerke für die Anbahnung von Technologie-Exportgeschäften überhaupt erst anzustoßen. Diese Funktion des BMK ist nach Einschätzung von österreichischen Teilnehmer*innen vor allem in Staaten bzw. auf Zielmärkten entscheidend, in denen die staatlichen 
Verwaltungen eine starke Rolle im Wirtschaftsgeschehen spielen, bzw. in Wirtschaftssektoren, die durch staatliche Akteure dominiert bzw. maßgeblich mitgestaltet werden. Dies ist vor allem bei Infrastrukturprojekten (z. B. Energie, Verkehr, Versorgung) der Fall, wobei damit auch alle dafür relevanten Schlüsseltechnologien (insbesondere auch Digitalisierung) ins Zentrum des Interesses rücken. Die Mehrzahl der Vertreter*innen der Unternehmen betonten in den Interviews, dass der Mehrwert der ATD-Veranstaltungen gegenüber anderen von AuBenwirtschaft Austria angebotenen Formaten im "quasi-offiziellen Charakter" der Veranstaltungen liegt. Dadurch würden Kontakte zu Schlüsselpersonen und Entscheidungsträgern in den Zielländern ermöglicht werden, die ohne Unterstützung durch das BMK nicht oder nur mit deutlich höherem Aufwand geknüpft werden können.

Den politischen Rahmen für ATD haben in der Vergangenheit häufig bestehende bilaterale Abkommen zwischen Österreich und den staatlichen Verwaltungen in den Zielländern gebildet. In den Gesprächen wurde die Bedeutung dieser bilateralen Abkommen bestätigt, um Räume für Technologieexport-Projekte zu eröffnen, einfachere Akquisitionswege zu schaffen und den Zugang zu internationalen Ausschreibungen zu verbessern. Der persönliche Kontakt der BMK-Vertreter vor Ort zu den Entscheidungsträgern in den Zielländern wurde in den Gesprächen ebenfalls als hilfreich erwähnt, um konkrete Projekte erfolgreich vorbereiten und abschließen zu können.

Im Lichte der Gespräche mit österreichischen ATD-Teilnehmer*innen ist der Ansatz des BMK, mit den TECXPORT-Veranstaltungen dort aktiv zu werden, wo ein Bedarf österreichischer Technologieanbieter oder für österreichische Technologie im Zielland artikuliert bzw. an das BMK herangetragen wird, richtig und zweckmäßig. Das wesentliche Alleinstellungsmerkmal des ATD-Formats ist strukturell mit der Rolle und Funktion des BMK als Türöffner verknüpft. Durch den direkten Zugang zu Schlüsselpersonen und Entscheidungsträgern werden für die österreichischen Technologieanbieter die Markterschließungswege verkürzt und vereinfacht. Die bilateralen politischen Kontakte des BMK geben den Unternehmen vor allem in der Frühphase zusätzliche Sicherheit und Unterstützung bei ihrem Engagement.

\section{Reisekostenzuschüsse zur Teilnahme an Austrian Technology Days (ATD)}

Reisekosten für die Teilnahme an Veranstaltungen zur Markterschließung im Ausland können zum Teil auch in anderen Förderprogrammen auf Bundes- und Landesebene mit gefördert werden. Außenwirtschaft Austria sieht hingegen für die Teilnahme an eigenen Veranstaltungen keine Reisekostenzuschüsse für die teilnehmenden Unternehmen vor. Im Gegenteil: Den Teilnehmer*innen wird ein geringer Teilnahmebeitrag verrechnet. Die Reise- und Aufenthaltskosten sind aus eigenen Mitteln zu tragen. Durch die Eigenfinanzierung der Kosten soll gewährleistet werden, dass die Unternehmen mit der Teilnahme an den Veranstaltungen tatsächlich ein wirtschaftliches Interesse demonstrieren. Die Kosten der Teilnahme an den Veranstaltungen stellen ein Investment dar, dass sich für die Unternehmen durch konkrete Internationalisierungsfortschritte und erfolgreiche Exportaktivitäten langfristig wirtschaftlich rechnen soll.

Da jedoch die ATD-Veranstaltungen im Rahmen der TECXPORT-Initiative sich grundlegend von anderen Auslandsreiseformaten unterscheidet - im Fokus von ATD steht die gezielte Ansprache von und Vernetzung mit Akteuren auf Auslandsmärkten zu spezifischen österreichischen Technologieangeboten - könnte aber durchaus argumentiert werden, dass Reise- 
kostenzuschüsse für die Teilnahme einzelner Unternehmen aufgrund der besonderen strategischen Ausrichtung der Veranstaltungen durchaus zweckmäßig sein können, sofern damit der Erfolg der Veranstaltungen insgesamt gesichert werden kann.

\section{TECXPORT-Plattform}

Mit der TECXPORT-Plattform wurde ein Kompetenzkatalog für österreichische Technologien erstellt, der aktuell mehr als 500 Einträge umfasst. Die TECXPORT-Plattform präsentiert österreichische Technologiekompetenzen in Form von Technologieprofilen auf eine ansprechende und kompakte Weise. Die Plattform kann auf Desktops und auf Mobilgeräten genutzt werden und steht potenziellen Nutzer ${ }^{*}$ innen sowohl als Online-App als auch als Offline-Version zur Verfügung.

Die TECXPORT-Plattform bietet den Nutzer*innen Informationen über österreichische Technologieangebote, die von Partnern in den Zielländern bzw. von potenziellen Kunden direkt genutzt oder durch österreichische Intermediäre an sie weitergegeben werden können. Die Informationen sollen dabei helfen, zielgerichtet passende österreichische Technologien für geplante Vorhaben in den Zielländern zu finden bzw. bekannt zu machen.

Andere Akteure in der österreichischen Förderungs- und Unterstützungslandschaft für die Technologie-Internationalisierung bieten keine inhaltlich oder funktional vergleichbaren Tools an. Auf der TECXPORT-Plattform werden nicht Unternehmen sondern Technologien präsentiert. Die TECXPORT-Plattform ist mit ihren Technologie-Profilen damit auch komplementär zum bestehenden Angebot der Außenwirtschaft Austria, die es österreichischen Anbietern ermöglicht, sich auf bestimmten Zielmärkten als Unternehmen zu präsentieren. TECXPORT eröffnet damit seinem spezifischen Technologiefokus einen zusätzlichen, alternativen Zugangsweg zu Informationen über österreichische Technologiekompetenzen, um die Sichtbarkeit für potenzielle Kunden zu erhöhen. Ein deutliches Alleinstellungsmerkmal hat die TECXPORT-Plattform aber insbesondere deshalb, weil sie sich explizit auch an die österreichischen Intermediäre für Technologie-Internationalisierung richtet und hier insbesondere die Arbeit der Wirtschaftsdelegierten und Technologie-Attachés in den österreichischen Auslandsvertretungen erleichtern und unterstützen soll. In den Gesprächen mit BMKMitarbeiter ${ }^{*}$ innen in den Technologiebüros in Peking und Jakarta und mit dem Wirtschaftsdelegierten des AußenwirtschaftsCentre Hongkong wurde dieser besondere Mehrwert der TEXCPORT-Plattform bestätigt.

\section{Innovationspolitischer Strukturbeitrag der TECXPORT-Initiative}

Schließlich leistet das BMK durch die operative Zusammenarbeit bei der Umsetzung der TECXPORT-Initiative mit der FFG und der Außenwirtschaft Austria einen sichtbaren Beitrag zur Umsetzung von Empfehlungen, die das Österreichische Wirtschaftsförderungsinstitut (WIFO) gemeinsam mit Consulting AG im Jahr 2015 im Zuge der Evaluierung von go-international an die verantwortlichen politischen Entscheidungsträger gerichtet hat. In der Evaluation stellten die Autor*innen fest, dass es in der österreichischen Förder- und Unterstützungslandschaft kaum Förderlücken gebe, jedoch ein großes Potenzial vorhanden wäre, die vorhandenen Angebote der verschiedenen Fördergeber auf Bundes- und Landesebene besser zu fokussieren und zu koordinieren.

Hervorzuheben ist hier insbesondere der strukturelle Mehrwert, der auf operativer Ebene durch die Zusammenarbeit zwischen FFG und Außenwirtschaft Austria entsteht. Die Außen- 
wirtschaft Austria und ihre Trägerorganisation WKÖ hat vielfältige und häufig intensive Kontakte zu bereits etablierten exportierenden bzw. exportorientierten österreichischen Unternehmen. Die FFG verfügt hingegen als nationale Förderagentur für anwendungsorientierte und unternehmensbezogene FuE über detaillierte Informationen über aktuelle technologische Entwicklungen bzw. über die daran beteiligten innovativen Unternehmen und angewandten Forschungseinrichtungen. Durch die Zusammenarbeit von FFG und Außenwirtschaft Austria werden die Stärken und spezifischen Kompetenzen der beiden Einrichtungen für eine effektive, synergistische Umsetzung von Maßnahmen zur Technologie-Internationalisierung miteinander verknüpft. 


\section{Relevanz und Nutzen für die Zielgruppen}

Die TECXPORT-Initiative adressiert mehrere Zielgruppen: Eine Zielgruppe sind die österreichischen Technologieanbieter, wobei sowohl gewerbliche Unternehmen als auch öffentliche Forschungseinrichtungen angesprochen werden. An diese Zielgruppe wenden sich die Instrumente Austrian Technology Days (ATD) und Reisekostenzuschüsse und indirekt auch das Instrument TECXPORT-Plattform. Die zweite Zielgruppe sind Intermediäre wie die Technologie-Attachés des BMK, die Wirtschaftsdelegierten an den AußenwirtschaftsCenters und sonstige mit Fragen des Technologieexports befasste Mitarbeiter*innen bei den österreichischen Botschaften im Ausland. Diese Zielgruppe wird als potenzielle Nutzer der TECXPORTPlattform angesprochen. Schließlich sind als Zielgruppe für die TECXPORT-Plattform auch Schlüsselpersonen und Entscheidungsträger in den öffentlichen Verwaltungen der Zielländer sowie potenzielle Kunden im Ausland angesprochen.

\subsection{Austrian Technology Days (ATD)}

In unseren Gesprächen mit Teilnehmer*innen an den ATD wurde deutlich, dass die österreichischen Unternehmen und Forschungsreinrichtungen die vorhandenen Unterstützungsangebote nur dann und in jenem Umfang in Anspruch nehmen, der für sie einen Mehrwert generiert. Wie wir bereits im vorhergehenden Abschnitt dargestellt haben, liegt für die Interviewpartner*innen der Mehrwert der ATD gegenüber anderen Veranstaltungsangeboten insbesondere darin, durch die Teilnahme Zugang zu hochrangigen Schlüsselpersonen und Entscheidungsträgern in der öffentlichen Verwaltung und in verwaltungsnahen Einrichtungen in den Zielmärkten zu erhalten. Es ist daher vor allem dieser Aspekt, der das TECXPORT-Angebot für österreichische Unternehmen und Forschungseinrichtungen interessant macht.

\section{Interview-Feedback-Box 1}

Ein großes österreichisches Technologieunternehmen, das an den ATD-Veranstaltungen in einem der TECXPORT-Zielländer teilnahm, konnte in diesem Jahr mehrere Großaufträge erfolgreich abschließen.

Für die erfolgreiche Realisierung der Geschäftsabschlüsse leistete nach Einschätzung des Unternehmens das BMK einen wichtigen Beitrag. Das BMK ermöglichte den Zugang zu Entscheidungsträgern in den Zielländern und nutzte seine politischen Kontakte, um die wirtschaftlichen Interessen österreichischer Unternehmen zu sichern. Für ein international agierendes Unternehmen im Infrastrukturbereich ist dies eine wichtige Funktion der öffentlichen Hand. In diesem Zusammenhang sind für das Unternehmen auch die bilateralen staatlichen Abkommen des BMK für den langfristigen Erfolg in der Exporttätigkeit sehr hilfreich. Gerade bei großen Infrastrukturprojekten braucht es Konstanz im Engagement vor Ort und einen langen Atem, um erfolgreich zu sein. Mit den ATD konnte den öffentlichen Stellen und Verwaltungen im Zielland gezeigt werden, was Technologie von Austria Inc. leisten kann.

Für die teilnehmenden Unternehmen und Forschungseinrichtungen stellen die ATD einen unter mehreren Bausteinen dar, die für die erfolgreiche Anbahnung und Realisierung von Kooperationsvorhaben und Technologieexportprojekten notwendig sind. Eine monokausale Zuordnung eines konkreten Technologieexporterfolges zu einer bestimmten ATD-Veranstaltung ist weder konzeptionell zu erwarten noch erwarten die teilnehmenden Unternehmen im 
Allgemeinen einen derartigen direkten bzw. unmittelbaren Erfolg. Trotzdem zeigen sich positive Wirkungen für die teilnehmenden Unternehmen. In den Interviews bestätigen mehrere Gesprächspartner*innen, dass durch ihre ATD-Teilnahme Kommunikationskanäle eröffnet und Kontakte in den Zielländern ermöglicht wurden, die das Weiterverfolgen der betrieblichen Internationalisierungsanstrengungen deutlich einfacher gemacht hat.

\section{Interview-Feedback-Box 2}

Ein österreichisches Kleinunternehmen im Bereich der Wasserqualitätskontrolle, das an einem ATD teilgenommen hatte, konnte im Zuge der Veranstaltung einige bereits Jahre zuvor geknüpfte Kontakte weiterführen und im Zuge der Veranstaltung auch neue Leads für Exportprojekte generieren. Im Zielland konnten bereits einige Anlagen gemeinsam mit einem europäischen Vertriebspartner, der über Niederlassungen im Zielland verfügt, in Betrieb genommen werden. Das Unternehmen arbeitet auch aktiv an der Vermarktung der Technologie für andere Zielländer. Dazu sind jedoch länderspezifisch weitere Entwicklungsarbeiten notwendig, die aus Eigenmitteln nur langsam umgesetzt werden können. Das Unternehmen nimmt daher heute schon die FuE-Förderungen der FFG in Anspruch, um rascher agieren zu können. Bessere Fördermöglichkeiten, um FuE-basierte Weiterentwicklungen von bestehenden Technologielösungen für neue Märkte erfolgreich abschließen zu können, würden das Unternehmen bei seinen Internationalisierungsbemühungen sehr unterstützen.

In jenen Fällen, in denen es bisher zu keinen nennenswerten Folgeaktivitäten oder konkreten Vermarktungserfolgen im Ausland gekommen ist, lag der Grund nicht im Design der Austrian Technology Days. Hauptsächlich wurden die Einschränkungen und Verzögerungen, die sich aufgrund der COVID-19-Epidemie ergeben haben, als Barrieren für das Weiterverfolgen der Kontakte in die Zielländer angegeben. In einigen Fällen gaben die Interviewpartner*innen auch an, sie würden nur über beschränkte interne Strukturen, personelle und finanzielle Ressourcen sowie Managementkapazitäten verfügen, um Internationalisierungs- und Exportprojekte voranzutreiben und wären daher noch zu keinen erfolgreichen Abschlüssen gekommen. Schließlich gaben Unternehmen an, dass gerade beim Zugang zu neuen Märkten, viele strategische Entscheidungen getroffen werden müssen, die sowohl rechtliche Aspekte betreffen können (z. B. Schutz des geistigen Eigentums) als auch die technische Gestaltung der vermarkteten Lösungen (z. B. Produkt oder Lizenzen) oder die Form des Marktzugangs (z. B. Tochtergesellschaft, lokale Vertriebspartner, Joint Venture) betreffen. Die Klärung dieser Fragen würde Zeit in Anspruch nehmen, um überhastete Entscheidungen zu vermeiden, die das Unternehmen möglicherweise langfristig auch schwächen könnten.

\subsection{ATD-Reisekostenzuschüsse}

Die Bedeutung der ATD-Reisekostenzuschüsse dürfte nach den Hinweisen unserer Gesprächspartner*innen eher gering sein. Die Kosten, die für einen Marktzugang auf internationalen Märkten insgesamt anfallen, sind hoch und Reisekosten machen darunter nur einen kleinen Teil aus. Durch die Förderung von Reisekosten wird daher im Allgemeinen keine additionale Wirkung erwartet werden können. Die strategische Entscheidung für ein neues Engagement auf einem Auslandsmarkt und die Sicherung der Finanzierung hierfür dürfte nicht von einem vergleichsweise geringen finanziellen Beitrag für die Teilnahme an einer Veranstaltung abhängig sein. 
Eine additionale Wirkung des Reisekostenzuschusses wäre nur in jenen Fällen gegeben, in denen sich eine Organisation erst infolge der Teilnahme an einem ATD dazu entscheidet einen Marktzutritt neu zu versuchen und damit in der Folge auch erfolgreich ist. Ein solcher zufälliger Erfolg durch eine ATD-Teilnahme stünde jedoch in einem gewissen Spannungsverhältnis zur BMK-Strategie, mit den TECXPORT-Aktivitäten im Normalfall nur dann tätig zu werden, wenn es einen konkreten, bereits an das BMK herangetragenen Bedarf österreichischer Technologieanbieter gibt.

Nur in jenen Fällen, in denen das BMK ohne bereits klar formulierte Nachfrage seitens österreichischer Technologieanbieter tätig wird (z. B. weil es Bedarf auf dem Zielmarkt gibt, der durch die Intermediäre vor Ort registriert wurde) könnte ein Reisekostenzuschuss ein Motivationsfaktor sein, um geeignete österreichische Technologieanbieter als Teilnehmer für eine ATD-Veranstaltung zu gewinnen.

Trotz der geringen zu erwartenden additionalen Wirkungen des Instruments sehen Unternehmen und außeruniversitäre Forschungseinrichtungen die Kostenzuschüsse der öffentlichen Hand sehr positiv, da sie in jedem Fall einen Deckungsbeitrag zu den Kosten ihrer Internationalisierungsanstrengungen leisten.

\subsection{TECXPORT-Plattform}

Primäre Zielgruppe der TECXPORT-Plattform sind die österreichischen Intermediäre in der Förderungs- und Unterstützungslandschaft für Technologie-Internationalisierung, die österreichische Technologieanbieter und potenzielle Kunden im Ausland zusammenbringen und vernetzen. Partner und Unternehmen im Ausland sind dann eine Zielgruppe der TECXPORTPlattform, wenn sie aufgrund ihrer Kontakte zu österreichischen Intermediären oder aufgrund der Teilnahme an ATD-Veranstaltungen an österreichischen Technologien interessiert sind. Informationen über die Nutzung durch österreichische Intermediäre und durch Dritte werden derzeit noch nicht systematisch erfasst und ausgewertet. Ein entsprechendes Monitoring ist jedoch in Vorbereitung.

\section{Interview-Feedback-Box 3}

Ein österreichischer Messtechnikhersteller wurde aufgrund des Technologieprofils auf der TEXCPORTPlattform zu einem virtuellen Workshop im Zielland eingeladen. In Folge dieser Veranstaltung erhielt das Unternehmen das Angebot, mit einem substanziellen öffentlichen Zuschuss des Ziellandes eine Auslandstochter zu gründen. Aufgrund strategischer, gesellschaftsrechtlicher und steuerrechtlicher Überlegungen entschied sich das Unternehmen schließlich für eine Minderheitsbeteiligung an einem JointVenture, das gemeinsam mit einem lokalen Partner gegründet wurde. Das Unternehmen ist auch in anderen Ländern der Region aktiv und arbeitet bei der Finanzierung von Projekten eng mit der Oesterreichischen Kontrollbank (OeKB) und mit der Oesterreichischen Entwicklungsbank (OeEB) zusammen. Das Unternehmen hat in der Vergangenheit bereits auch die Möglichkeit genutzt, über die Austrian Development Agency (ADA) eine Machbarkeitsstudie zu realisieren.

In unseren Gesprächen mit Intermediären wurden der inhaltliche Ansatz (d. h. Fokus auf Technologien bzw. Technologieangebote), die Gestaltung und die potenziellen Nutzungs- 
möglichkeiten der TECXPORT-Plattform als sehr positiv bewertet. Die Gesprächspartner*innen wiesen darauf hin, dass neben einer Nutzung der TECXPORT-Plattform durch österreichische Intermediäre ein wesentlicher Mehrwert auch durch die Weitergabe von PlattformInhalten an Intermediäre in den Zielmärkten (d. h. Ansprechpartner*innen in den öffentlichen Verwaltungen der Zielländer) erreicht werden kann bzw. in Zukunft erreicht werden könnte. Inzwischen würden die Inhalte der Technologieprofile und die sprachliche Qualität der Übersetzungen ein Niveau haben, das die Weitergabe der Informationen an Dritte möglich mache. Außerdem betonten einige Gesprächspartner*innen, dass die Zugänglichkeit der TECXPORTPlattform als mobile App und die offline-Funktionalität vor allem für Märkte in Asien ein wichtiges Feature sei.

Die Intermediäre sehen in der TECXPORT-Plattform ein Instrument mit einem durchaus interessanten Entwicklungspotenzial. Die TECXPORT-Plattform könnte als Unterstützungstool dienen, um beispielsweise rasch auf konkrete Anfragen von Kontaktpartner*innen in den Zielländern nach möglichen österreichischen Technologieanbietern reagieren zu können, oder auch beim Vorbereiten von themenbezogenen Veranstaltungen, beispielsweise um einen ersten Überblick über österreichische Akteure in den verschiedenen technologischen Themenfeldern zu erhalten. Voraussetzung für eine effiziente Nutzung sei neben einer einfachen Bedienbarkeit und einer zielführenden Suchfunktion, die Qualität und Aktualität der gebotenen Informationen zu den Technologieprofilen und über die hinter den Profilen stehenden Unternehmen bzw. Technologieanbieter.

\section{Interview-Feedback-Box 4}

Ein österreichischer Spezialfahrzeugausrüster, der bereits seit vielen Jahren auf dem Zielmarkt tätig ist und auch über ein mit fünf Mitarbeiter*innen ausgestattetes Repräsentationsbüro vor Ort verfügt, steht regelmäßig mit den Mitarbeiter*innen des AußenwirtschaftsCenters vor Ort in Kontakt. Auch ein Technologie-Attaché des BMK ist vor Ort unterstützend tätig. Für das Unternehmen ist die wichtigste Leistung der österreichischen Auslandsvertretung, frühzeitig Informationen über geplante öffentliche Beschaffungsprozesse der Gebietskörperschaften im Zielland bereitzustellen. Hierbei spielt die BMK-Vertretung vor Ort eine wichtige Rolle, da sie häufig in direktem Kontakt zu den ausschreibenden Behörden steht. Die bereitgestellten Informationen und Kontakte ermöglichen es dem Unternehmen frühzeitig planerisch aktiv zu werden. Dies ist vor allem deshalb für das Unternehmen sehr wichtig, weil für jedes Projekt besondere lokale Verfahren gelten und spezifische technische Vorgaben und Anforderungen an die angebotenen Lösungen berücksichtigt werden müssen. Eine besondere Herausforderung für das Unternehmen stellen Finanzierungsfragen dar. Zahlreiche Leads würden scheitern, weil keine für die beschaffenden Gebietskörperschaften geeigneten bzw. überzeugenden Finanzierungsmodelle gefunden werden können.

Für unsere Gesprächspartner*innen war der Fokus auf die Zielgruppe Intermediäre nachvollziehbar und zweckmäßig. Um das Angebot beispielsweise auch für Kunden im Ausland direkt nutzbar und attraktiv zu machen, wäre eine andere, noch viel stärker auslandskundenfokussierte Darstellung der Technologieprofile gefordert. Würde man dies tatsächlich wollen, müssten Änderungen bereits bei der Namensgebung der Plattform beginnen. Potenzielle ausländische Kunden wären ja nicht am Technologieexport Österreichs interessiert, sondern an konkreten innovativen Technologielösungen für ihre spezifischen Anforderungen oder 
Problemstellungen in den Zielländern. Die Einschätzungen unserer Gesprächspartner ${ }^{*} i n n e n$ zur Gestaltung und zum Nutzungspotenzial der TECXPORT-Plattform legen den Schluss nahe, dass für die breitere Nutzung der Plattform durch potenzielle Kunden im Ausland sofern dies durch das BMK in Zukunft überhaupt angestrebt werden sollte - eine effektivere Darstellung und Verknüpfung der Technologieprofile auf der Plattform mit einer Präsentation österreichischer Unternehmen und Österreichs als leistungsfähiges Technologiekompetenzland notwendig wäre.

Die österreichischen Technologieanbieter stellen die Informationen zur Verfügung, die als Grundlage für die Gestaltung der Technologieprofile auf der TECXPORT-Plattform dienen. In den Gesprächen mit den Technologieanbietern haben wir unsere Gesprächspartner*innen danach gefragt, wie sie den Nutzen der Plattform für die Umsetzung konkreter Vorhaben (z. B. Exportprojekte) einschätzen. Der überwiegende Anteil unserer Gesprächspartner*innen sieht in der TECXPORT-Plattform nur einen geringen direkten Nutzen. Ein großer Teil der Gesprächspartner*innen verfolgt die eigenen Beiträge zur TECXPORT-Plattform auch nur mit geringer Aufmerksamkeit. Die Gesprächspartner*innen wiesen wiederholt darauf hin, dass aus ihrer Perspektive die Ermöglichung persönlicher Kontakte und die Vernetzung mit Partnern im Ausland deutlich effektiver für die Realisierung von konkreten Projekten sei als Informationsangebote über Technologie-Know-how, egal ob Print oder in digitaler Form.

Mehrere Gesprächspartner*innen waren der Ansicht, dass die TECXPORT-Plattform in ihrer heutigen Form für potenzielle Kunden keinen nenneswerten Mehrwert gegenüber einer eigenen Webrecherche hätte. Trotzdem wäre die Plattform für österreichische Anbieter aber ein zusätzlicher Marketingweg, der neben anderen Formen der Darstellung und Kommunikation der eigenen Technologieangebote genutzt werden kann.

Auch wenn Kunden im Ausland ohnehin keine unmittelbare Zielgruppe der TECXPORT-Plattform sind, können die Rückmeldungen aus den Interviews doch auch einen Ansporn geben, bei der Weiterentwicklung der TEXCPORT-Plattform noch stärker auf jene Aspekte zu achten, die konzeptionell den Mehrwert gegenüber Webrecherchen und Internet-Auftritten von Unternehmen sichern sollen: Die TECXPORT-Plattform verzichtet auf umfangreiche Firmenpräsentationen, sondern stellt Technologielösungen und Technologiekompetenzen kurz und prägnant dar. Die TECXPORT-Plattform erlaubt einen Einstieg und einen Überblick über österreichische Technologieangebote, die über eine eigene Webrecherche hinsichtlich Dichte, Qualität und Sprachangebot nicht erreicht werden kann. 


\section{Empfehlungen zur zukünftigen Ausrichtung}

Aus den Hinweisen in den Gesprächen mit den Unternehmen und Forschungseinrichtungen zu den Aktivitäten, Ergebnissen und Wirkungen der bisherigen Maßnahmen der TECXPORTInitiative ziehen wir den Schluss, dass es dem BMK mit seinen Partnern insgesamt gelingt, österreichische Technologieanbieter dabei zu unterstützen, einfacher Zugang zu internationalen Märkten zu erhalten. TECXPORT erleichtert Unternehmen und anderen Technologieanbietern den Weg zu konkreten Vermarktungsprojekten. Die Stärke und das Alleinstellungsmerkmal der TECXPORT-Initiative liegen vor allem in den besonderen Möglichkeiten, die das BMK den Unternehmen und Forschungseinrichtungen durch seine Türöffnerfunktion bzw. durch das Vor-Ort-Engagement der BMK-Mitarbeiter*innen und seiner Kooperationspartner bieten kann. Der quasi-offizielle Charakter der ATD-Veranstaltungen wird durch den Verweis bzw. die Bezugnahme auf bestehende bilaterale Abkommen zwischen Österreich und den Zielländern noch unterstützt. Damit eröffnen sich für österreichische Technologieanbieter neue Handlungs- und Gestaltungsräume für Kooperationen und Geschäftsanbahnungen. Das BMK ist in TECXPORT-Veranstaltungen in einer Form tätig, die aufgrund des offiziellen, ministeriellen Charakters deutlich über die Möglichkeiten von Außenwirtschaft Austria hinausgehen. Die österreichischen Technologieanbieter sehen daher gerade in diesem Aspekt den besonderen Mehrwert des TECXPORT-Angebots.

\subsection{Empfehlungen zur Weiterentwicklung der Förderinstrumente}

Für die zukünftige Ausrichtung der TECXPORT-Instrumente lassen sich aus den empirischen Ergebnissen der Konzeptevaluierung die folgenden Schlüsse ziehen:

Konzept der Austrian Technology Days (ATD) konsequent weiterverfolgen

Bei der Vorbereitung und Ausrichtung von ATD sollte das BMK weiterhin sehr klar auf die Unterstützung konkreter Bedarfe fokussieren, die entweder aktuelle innovations-und technologiepolitische Schwerpunkte des Ministeriums adressieren oder die sich auf die bilateralen ministeriellen Abkommen über die Technologiezusammenarbeit zwischen Österreich und anderen Staaten in internationalen Wachstums- und Zukunftsmärkten beziehen. Die Bedarfe können angebotsseitig durch österreichische Technologieanbieter an das BMK herangetragen werden oder nachfrageseitig durch die BMK-Technologieattachés bzw. durch die Partner in den österreichischen Auslandsvertretungen aufgrund von Kontakten mit potentiellen Kunden und Politikverantwortlichen vor Ort formuliert werden.

Von besonderer Bedeutung für ATD sind dabei jene Technologiebereiche und internationalen Kooperationen zur Bewältigung von globalen Herausforderungen, für die das BMK als Technologie-, Innovations- und Infrastrukturministerium sachlich zuständig ist bzw. bereits in der Vergangenheit Transferaktivitäten entwickelt und unterstützt hat: Energie, Mobilität, Umwelt, Gesundheit, Industrielle Technologien, Luft- und Raumfahrt, IKT und (urbane) Infrastruktur und Sicherheit bleiben hier die zentralen Themenfelder.

Bei den ATD handelt es sich um strategische Veranstaltungen, die konkrete, durch das BMK im Rahmen seiner hoheitsstaatlichen Funktionen unterstützte Initiativen zur Förderung von gezielten Technologiekooperationen zwischen österreichischen Anbietern und Partnern in 
den ausgewählten Zielländern verfolgen. Die ATD-Veranstaltungen können wie bereits erwähnt entweder bei formulierten Bedarfen österreichischer Technologieanbieter ansetzen oder eine konkrete Nachfrage auf den Zielmärkten nach österreichischen Lösungen adressieren. Dazu lädt das BMK gezielt österreichische Teilnehmer*innen zu den ATD-Veranstaltungen ein, um genau jene österreichischen Akteure vor Ort zu haben, die ein dem Bedarf im Zielland entsprechendes Angebot vorweisen können. Mit diesem Konzept sollte gewährleistet sein, dass die teilnehmenden Unternehmen und Technologieanbieter über die strukturellen Ressourcen (d. h. Technologieangebote, Personal, Managementkapazitäten) und die finanzielle Leistungsfähigkeit verfügen, die Voraussetzung für die erfolgreiche Anbahnung und den Abschluss von Technologieexportgeschäften bzw. nachhaltigen bilateralen Geschäftsbeziehungen sind.

\section{ATD-Reiskostenzuschüsse strategisch einsetzen und fokussieren}

Aufgrund der zu erwartenden geringen additionalen Wirkung sollte die Zweckmäßigkeit der Reisekostenzuschüsse für TECXPORT-Veranstaltungen grundsätzlich überdacht werden. Durch Änderungen bei der Abwicklung von Förderungen für Kleinstprojekte (wozu auch die ATD-Reisekostenzuschüsse zählen) konnten die in der Vergangenheit hohen administrativen Kosten des Instruments bereits deutlich verringert werden.

Sollte das BMK die Möglichkeit von Reisekostenzuschüssen weiterhin vorsehen wollen, sollte das Instrument möglichst strategisch eingesetzt werden und sich auf Teilnehmer*innen konzentrieren, bei denen eine additionale Wirkung der Förderung erwartet werden kann. Reisekostenzuschüsse könnten für die Teilnahme von Technologieanbietern vorgesehen werden, wenn deren Anwesenheit aus Sicht des BMK einen substanziellen Beitrag zum Gesamterfolg der Veranstaltung im Ausland leistet.

Die ATD-Veranstaltungen selbst könnten für die Teilnehmer*innen weiterhin kostenlos bleiben. Durch das Tragen der eigenen Reise- und Aufenthaltskosten würden die Teilnehmer*innen ihr strategisches Interesse an einer Wirtschaftskooperation in den Zielländern glaubhaft demonstrieren und die Bereitschaft, dafür auch die notwendigen personellen und finanziellen Ressourcen zu investieren.

\section{TECXPORT-Plattform als Unterstützungstool für Intermediäre positionieren}

Für die Fortführung der TECXPORT-Plattform schlagen wir vor, das Nutzer- bzw. Nutzungskonzept weiter zu schärfen und zu fokussieren. Die Hinweise aus den Gesprächen mit den Vertreter ${ }^{*} i n n e n$ von österreichischen Technologieanbietern und Intermediären bestätigen, dass das TECXPORT-Angebot in seiner derzeitigen Form vor allem als Informations- und Unterstützungstool für österreichische Intermediäre im In- und Ausland einen Mehrwert leisten kann.

In diesem Zusammenhang sollte geprüft werden, ob und in welcher Form eine stärkere Vernetzung des TECXPORT-Angebots mit internen Informations- und Kommunikationsinstrumenten von Außenwirtschaft Austria zweckmäßig wäre, um die Verbreitung und Nutzung von TECXPORT in der Zielgruppe der Intermediäre zu unterstützen. Die Technologieprofile, die unter anderem auf aktuellen Forschungsergebnissen und Innovationsprojekten von Unternehmen und außeruniversitären Forschungseinrichtungen beruhen (die zum Kundenkreis der FFG für FuE-Förderungen gehören), könnten einen wichtigen Informationsmehrwert für die Wirtschaftsdelegierten und Technologie-Attachés insgesamt bieten. Durch die Stärkung des Austausches zwischen FFG und Außenwirtschaft Austria über die TECXPORT-Plattform 
würde das BMK einen Beitrag zur Steigerung der Wirkungen der öffentlichen FuE-Förderung im Rahmen der Technologie-Internationalisierung leisten. Die FFG hat bereits in der Vergangenheit Schritte in diese Richtung gesetzt, beispielsweise in Form von Webinaren für Wirtschaftsdelegierte. Diese Maßnahmen sollten weitergeführt und verstärkt werden.

Neben dem technischen, gestalterischen und Nutzungs-Design der TECXPORT-Plattform wurde in den vergangenen Jahren insbesondere auf die Ausweitung des Angebots an Technologieprofilen geachtet. Es steht außer Frage, dass eine kritische Masse an Technologieprofilen die Voraussetzung für eine Nutzung durch die adressierten Zielgruppen ist. Gleichzeitig muss sichergestellt werden, dass die Qualität und Aktualität der Einträge eine Nutzung bzw. eine Weitergabe von Informationen aus der Plattform an Dritte erlaubt. Nutzerfreundlichkeit (z. B. Suchfunktionen, Verlinkungen, Downloads von Informationen) und leichte Integration in die Arbeitsroutinen der adressierten Zielgruppen sind ebenfalls wichtige Elemente für eine erfolgreiche Inanspruchnahme der Plattform. Die FFG hat in ihren Arbeitsprogrammen für die Jahre 2020 und 2021 auf diese Aspekte bereits Bezug genommen und setzt entsprechende qualitätsfördernde Maßnahmen derzeit um.

\section{Bei neuen FTI-Förderinstrumenten auf die Erfahrungen der FFG aufbauen}

Das BMK arbeitet aktuell an der strategischen Weiterentwicklung der TECXPORT-Initiative. Angedacht ist eine Erweiterung des Instrumentenportfolios um bilaterale, auslandsmarktfokussierte FTI-Kooperationen zur gezielten Positionierung österreichischer Technologieanbieter in ausgewählten Zielländern mit dem Ziel der Verwertung der Lösungen im Ausland. Darüber hinaus ist unter dem Arbeitstitel "Tailored Innovation" ein zusätzliches Instrument in Planung, um durch eine marktspezifische Anpassung von Produkten und Lösungen im Rahmen von Kunden-Kooperationen neue Märkte für österreichische Partner aufzuarbeiten.

In den Interviews mit den Unternehmen und Forschungseinrichtungen haben unsere Gesprächspartner*innen deutlich zum Ausdruck gebracht, dass sie ergänzende FTI-Förderinstrumente als zweckmäßig, attraktiv und zielführend einschätzen, um einen erfolgreichen Marktzugang auf neuen Exportmärkten zu unterstützen. Fast alle unsere Gesprächspartner*innen gaben an, dass zusätzliche Fördermöglichkeiten für bilaterale FTI-Kooperationen mit Partnern im Ausland bzw. für die Entwicklung von Anpassungsinnovationen für spezifische Zielmärkten ein sehr interessantes Unterstützungsmodell seien.

Bei der Positionierung, Gestaltung und Abwicklung von derartigen zusätzlichen Förderangeboten sollte auf eine optimale Einbettung in das bereits bestehende Förderumfeld geachtet werden. Die FFG Bereiche Basisprogramme, Thematische Programme und Strukturprogramme bieten bereits heute zahlreiche Ausschreibungsformate an, die internationale, bilaterale oder transnationale Kooperationen und / oder Internationalisierungsanstrengungen (mit) unterstützen.

Bei der Gestaltung der Förderangebote sollte jedenfalls auf die bereits vorliegenden Erfahrungen der FFG bei der Vorbereitung und Abwicklung bilateraler, transnationaler und internationaler Ausschreibungen und Förderprogramme (z. B. Beyond Europe, ERA-Net-Initiativen, Eureka und Eureka-Cluster, internationale Aktivitäten im Rahmen der EU-Rahmenprogramme, internationale Ausschreibungen im Rahmen thematischer BMK-Programme) aufgebaut werden. Bevorzugt sollten die bereits vorhandenen Abwicklungsstrukturen in der FFG genutzt werden. Auf eine Duplizierung von Programmformaten und der dazu notwendigen Abwicklungsstrukturen sollte verzichtet werden, um administrative Effizienz und größtmöglichen Nutzen für die Zielgruppen zu gewährleisten. 
Instrumente und Verfahren für FTI-Förderungen auf Förderbudgets abstimmen

Insbesondere sollten die angebotenen FTI-Förderinstrumente auf das zur Verfügung stehende Förderbudget abgestimmt werden. Die Ausschreibungen sollten einerseits einen Wettbewerb zwischen den Antragstellern zulassen, andererseits aber gleichzeitig auch eine ausreichende Förderwahrscheinlichkeit von qualitativ hochwertigen Anträgen im Sinne der Förderzielsetzungen sicherstellen. Schließlich sollte der administrative Aufwand der Förderstelle und jener der Antragsteller in einem vertretbaren Verhältnis zum Förderbudget bzw. zum erwarteten, mit der Förderung stimulierten Gesamtvolumen an direkten und indirekten Aktivitäten bei den geförderten Partnern stehen.

\subsection{Empfehlungen zur Gestaltung der Prozesse der TECXPORT-Initiative}

Dort tätig werden, wo das BMK als Türöffner den Unterschied macht

Die TECXPORT-Initiative sollte weiterhin primär und konsequent an der Schnittstelle von politischen Schwerpunkten zur Technologie-Internationalisierung des BMK und den aktuellen Bedarfen von österreichischen Technologieanbietern bzw. den erwarteten Chancen (auf Basis des spezifischen Bedarfs im Zielland) für österreichische Technologieanbieter auf internationalen Wachstums- und Zukunftsmärkten ansetzen. Bei der Zusammenarbeit mit österreichischen Technologieanbietern sollte im Auge behalten werden, dass es oft nicht an fehlenden Förder- und Unterstützungsangeboten liegt, die den erfolgreichen Export von Technologien hemmen, sondern an mangelnden Strukturen und fehlenden Managementkapazitäten in Unternehmen und Forschungseinrichtungen. Auch rechtliche Aspekte und Finanzierungsfragen beeinflussen die Realisierbarkeit und die Geschwindigkeit eines unternehmerischen Exportprojekts. Das BMK kann in erster Linie als Türöffner auftreten und sollte diese Funktion weiter sehr aktiv wahrnehmen.

\section{Weiter auf Kooperation setzen, um Synergien zu sichern}

Effektivität und Effizienz von Förder- und Unterstützungsmaßnahmen werden auch durch den Grad an Abstimmung und Vernetzung der Angebote der verschiedenen involvierten Einrichtungen beeinflusst. Das BMK hat durch die Zusammenarbeit mit der FFG und Außenwirtschaft Austria diesen Aspekt erkannt und im Design der TECXPORT-Initiative bereits adressiert. Die Konzeptevaluierung hat zudem gezeigt, dass das BMK durch die Zusammenarbeit zwischen FFG und Außenwirtschaft Austria in der TECXPORT-Initiative Synergien schafft. Auf diese Dimension des Programms sollte in Zukunft noch stärker geachtet werden.

Ziele für die Initiative formulieren, die Aussagen über den Erfolg der Maßnahmen zulassen und die mit den Instrumenten direkt adressiert werden können

Bisher wurden die Ziele der TECXPORT-Initiative vor allem dahingehend formuliert bzw. kommuniziert, dass durch die Maßnahmen österreichische Technologieanbieter direkt zu konkreten Vermarktungserfolgen kommen sollen. Im Lichte der Ergebnisse unserer Gespräche mit Unternehmen und Forschungseinrichtungen halten wir dieses Ziel mit den eingesetzten Förderinstrumenten nicht unmittelbar adressierbar und damit auch für nicht realistisch. Die TECXPORT-Veranstaltungen, die Reisekostenzuschüsse und die TECXPORT-Plattform bilden in den meisten Fällen einen von zahlreichen Bausteinen auf dem Weg zu einem erfolgreichen Technologieexportprojekt bzw. einer nachhaltigen Technologie-Kooperation 
mit Partnern im Ausland. Die Zeithorizonte für konkrete Vermarktungserfolge können in einzelnen Fällen kurz sein, in anderen Fällen hingegen jedoch auch mehrere Jahre betragen. Außerdem bleibt ein wichtiger Aspekt des TECXPORT-Programms, nämlich die damit erzielte Vernetzung der Förderangebote bzw. der Aktivitäten unterschiedlicher Akteure (insbesondere zwischen BMK, FFG und Außenwirtschaft Austria) in der bisherigen Zielformulierung ganz ausgeblendet.

Wir schlagen daher vor, sowohl für die einzelnen Förderinstrumente als auch für die Programmprozesse adressierbare Ziele und Indikatoren zu formulieren, die eine Beurteilung des laufenden Programmerfolgs mit Blick auf die eingesetzten Förderinstrumente und die Programmprozesse in quantitativer und / oder qualitativer Form erlauben. Am einfachsten dürfte dies für die zukünftigen geplanten FTI-Förderinstrumente fallen, da für FFG-geförderte FuE-Vorhaben bereits ein Monitoring der Projektwirkungen besteht bzw. auf dieses aufgebaut werden kann.

\section{Monitoring-System für Governance und Programmsteuerung stärken}

Die Governance und Programmsteuerung der TECXPORT-Initiative sollte durch ein strukturiertes Monitoring-System über die Leistungen, Ergebnisse und Wirkungen der Programmprozesse sowie der eingesetzten Instrumente unterstützt werden. Die bereits bestehenden Ansätze hierzu im Rahmen der jährlichen Programmplanung und Berichterstattung sollen weiterentwickelt werden.

Mit Blick auf die Ergebnisse und Wirkungen der TECXPORT-Veranstaltungen bieten die derzeit bereits durch die FFG durchgeführten Feedback-Befragungen der ATD-Teilnehmer*innen einen zweckmäßigen Ausgangspunkt. In diesem Zusammenhang halten wir auch die von der FFG bereits durchgeführte telefonische Nachverfolgung der Kontakte und die Dokumentation der Folgeaktivitäten der Teilnehmer*innen drei bzw. zwölf Monate nach der Veranstaltung für einen wichtigen und richtigen Ansatz, um Hinweise für die Gestaltung der Veranstaltungen zu erhalten, und um mehr über die fördernden und hemmenden Faktoren für erfolgreiche Kooperationen und Geschäfte im Nachgang der Veranstaltungen zu erfahren.

Bezüglich des Erfolgs und der Wirkungen der TECXPORT-Plattform sollten die Ziele und die Zielindikatoren in erster Linie auf die Potenziale und die konkrete Nutzung durch die intermediären Akteure abzielen: Wie entwickeln sich die Nutzerzahlen und Nutzungsintensität unter österreichischen Intermediären? Für welche Aufgaben oder Zwecke nutzen Intermediäre die Plattform? Entsprechen Umfang und Zwecke der Nutzung den gesetzten Zielen und Erwartungen?

Weitere qualitative Informationen, die für die Programmsteuerung von Interesse wären, sind bespielweise dokumentierte Hinweise von Intermediären und ihren Ansprechpartner*innen im Ausland über die Rezeption der Förderinstrumente in den Zielländern. Dieses Feedback sollte für die laufende interne Erfolgskontrolle und für die Weiterentwicklung der Instrumente des TECXPORT-Programms aktiv genutzt werden. 
inspiperesearch

inspire research Beratungsgesellschaft m.b.H.

Lange Gasse 65/16 1080 Wien 